\title{
Improving the Success of Mailed Letter Intervention Programs to Influence Prescribing Behaviors: A Review
}

\author{
Mei-Jen Ho, PharmD, MSPH, and Jineane Venci, PharmD
}

\begin{abstract}
BACKGROUND: Educational interventions have long been used as a means of influencing prescribing behavior. Various techniques including educational mailings, academic detailing, prescriber feedback with or without disclosing patient-identifying data, and supplemental patient information have been used to promote appropriate prescribing habits, reduce costs, and optimize patient care. While the effects of educational intervention programs are widely reported, little information is available regarding the effectiveness of various mailed intervention techniques.
\end{abstract}

OBJECTIVE: To review the effectiveness of mailed intervention programs and identify factors that may promote successful outcomes.

METHODS: A literature search was conducted via PubMed for reports of mailed intervention programs published through May 2012. Specific search terms included "drug utilization review," "drug utilization," "Medicaid," "prescribing feedback," "mailed physician intervention," and "mailed physician communications." Identified publications that met the following criteria were selected for inclusion: (a) evaluated printed educational materials disseminated via postal mail, (b) occurred in an outpatient setting, and (c) measured intervention impact on prescribing patterns, health care utilization, or economic outcomes. Publications that met all 3 criteria were abstracted for intervention strategy, follow-up period, data source, intervention target, prescriber acceptance of intervention, and effect on prescribing patterns, health care utilization, and economic outcomes.

RESULTS: A total of 40 published reports regarding 39 unique interventions met inclusion criteria. The majority (34/39 [87.2\%]) of studies were conducted in state or federally funded programs; only 5 programs involved private insurers. All programs used follow-up periods of $\leq 12$ months after final intervention mailing. A total of 26 of the 39 unique interventions reported a positive impact on at least 1 target outcome. Programs that included a second recipient such as pharmacists $(n=4)$ reported a greater impact as compared with interventions mailed to prescribers alone. Programs that provided patient-identifying data had a higher success rate than those that supplied prescriber feedback and/or educational materials $(21 / 25$ [84.0\%] vs. $5 / 14$ [35.7\%]); it should be noted that 2 of the 5 successful programs that provided nonpatient-identifying materials also used academic detailing. Programs that sent education material and/or prescriber feedback pertaining to multiple medication classes or disease states had minimal impact on prescribing patterns $(n=4)$. However, targeting 1 specific disease or medication supported by appropriate evidence resulted in favorable change in a short period of time. Additionally, providing recommendations that were supported by widely accepted clinical guidelines or literature were also associated with a high rate of success. A subset of programs that sought to evaluate health care utilization $(n=5)$ and economic impact $(n=9)$ observed little change in measured outcomes. Evaluation of prescriber response forms conducted by 7 programs revealed that changes in therapy occurred in approximately $50 \%$ of patients with prescribers who intended to accept intervention recommendations.

CONCLUSIONS: Though the degree of heterogeneity between articles prevents provision of definite results, it appears that a well-constructed mailed intervention program has the potential to evoke significant changes in prescribing patterns. Prescribers appear to be receptive to mailed interventions; however, there are limited data to determine the association between acceptance and actual prescribing change. Future research should focus on identifying barriers that may prohibit acceptance of recommendations from translating into changes in therapy. Additionally, future projects should include longer assessment periods to determine the duration of impact following final intervention mailing and potential effect on health care and economic outcomes.

J Manag Care Pharm. 2012;18(8):627-49

Copyright $\odot 2012$, Academy of Managed Care Pharmacy. All rights reserved.

\section{What is already known about this subject}

- Retrospective drug utilization review is widely used by thirdparty payers and required of state Medicaid agencies to ensure the appropriateness of prescription drug use. Mailed letter interventions are often used to alert prescribers of potentially inappropriate prescribing, communicate therapeutic recommendations, and provide notification of potential for adverse effects. Intervention letters are assumed to have a direct effect on treatment of identified patients as well as a "spillover" effect onto other patients under the prescriber's care.

- Previous publications evaluating the effectiveness of multifaceted interventions have focused heavily on the use of academic detailing or other forms of live educational outreach. Evaluations of mailed intervention programs alone have produced mixed results, and little information is available regarding the comparative effectiveness of various mailed intervention strategies.

- Figueiras et al. (2001) noted interventions that engage prescribers in personal contact ("active intervention") are more effective than those that provide unsolicited mailed materials ("passive intervention"). The authors concluded that combining active and passive strategies and formulating materials to be personalized to the recipient appeared to be the most effective intervention design. 


\section{What this review adds}

- This subject review discusses the overall effectiveness of mailed intervention programs published through May 2012 and identifies factors that may contribute to prescriber acceptance.

- Approximately $67 \%$ of identified programs reported a successful change in at least 1 measured outcome, including changes in prescribing patterns, health care utilization, and economic effects. Key factors associated with intervention success include (a) provision of patient-identifying data, (b) selection of recipients based on prescribing habits, (c) inclusion of a second intervention recipient, and (d) provision of focused recommendations that can be supported by widely accepted clinical guidelines or literature.

- Recipient response forms generally indicated acceptance of intervention programs. However, many programs reported only $50 \%$ of patients with a prescriber who indicated agreement with intervention recommendations had an actual change in therapy upon follow-up review.

$\mathrm{U}$ nder the Omnibus Budget Reconciliation Act of 1990 (OBRA 90), state Medicaid programs are mandated to conduct drug utilization reviews to ensure beneficiaries are receiving safe and appropriate therapy. ${ }^{1}$ Beginning in 1993, state Medicaid agencies were required to conduct retrospective drug utilization reviews on outpatient medications to identify potential overuse or unnecessary medication therapy. Since this time, the use of retrospective drug utilization review has expanded to health maintenance organizations (HMO), Medicare programs, Veterans Administration (VA) medical centers, and various health management programs outside of the United States. ${ }^{2-7}$ In addition to promoting safe and effective therapy, retrospective drug utilization review can also be used to coordinate care among physicians, reduce medication costs, and promote changes in prescribing behavior to reflect evidence-based recommendations. ${ }^{8-12}$

Medication-related concerns identified during retrospective drug utilization reviews may be communicated to prescribers using mailed intervention letters. Materials contained in intervention packets vary among programs, often according to targeted outcomes and/or availability of data, and may include patient-identifying data, prescriber feedback, educational information, and supplemental patient education materials. Although every program appeals to its stakeholders because the purpose of retrospective drug utilization reviews is to provide appropriate therapeutic coverage at the lowest possible costs, external reviewers may be driven by incentives to reduce costs for the parent organization and providing strategies focusing only on short-term savings. In the United States, patient-identifying information (i.e., patient names and/or prescription claims histories) are frequently provided in accordance with the Health Insurance Portability and Accountability
Act (HIPAA) $)^{2-4,8-25}$; provision of such information is uncommon among programs conducted overseas. Programs outside of the United States commonly use prescriber feedback alone or in combination with educational materials to encourage changes in prescribing practices., ${ }^{5,626-30}$ Printed materials are often used in combination with academic detailing or other forms of educational outreach to create a multifaceted educational intervention. ${ }^{26,31,32}$

The impact of educational intervention programs and retrospective drug utilization review has been widely reported in the literature. ${ }^{33-37}$ Intuitively, we expect that unsolicited printed materials would improve prescribing patterns, but it is difficult to quantify its association to improved clinical outcomes or health care resource costs. Previous systematic reviews reported that passive interventions, such as including unsolicited mailings, are not as effective as active interventions, such as academic detailing..$^{34,38-42}$ No previously published subject reviews have examined the comparative effectiveness of various types of printed materials alone. The purpose of this review is to evaluate the effectiveness of mailed intervention programs that used primarily printed techniques and identify factors that have been associated with successful outcomes.

\section{Methods}

A comprehensive PubMed search was conducted in May 2012 using the following search terms: "drug utilization review," "drug utilization," "Medicaid," "prescribing feedback," "mailed physician interventions," and "mailed physician communications." No search limitations were set to include practices outside of the United States, as the purpose of this review was to identify factors that may influence prescribing behaviors. The search strategy is summarized in Figure 1.

Titles and abstracts were screened to identify studies that met the following inclusion criteria: (a) evaluated an educational intervention program in which at least 1 study group received only printed materials disseminated via postal mail, (b) occurred in an outpatient setting, and (c) aimed to measure intervention impact on prescribing patterns, health care utilization, or economic outcomes. After the initial screen, both authors reviewed the objectives and methods of identified articles to ensure each publication fulfilled the screening criteria.

During the full review, programs that exclusively measured impact of interventions mailed to nonprescribing practitioners, such as pharmacists or nurses, were excluded. Programs that specified inclusion of both physician and midlevel prescribers were included. Interventions provided via fax, e-mail, computerized physician reminders, patient chart, or verbal communication were excluded, as the purpose of this review was to evaluate the value of delayed recommendations. Publications that involved, but did not directly evaluate, the impact of a mailed intervention were also excluded. Additionally, the reference lists were manually reviewed for potential publications 


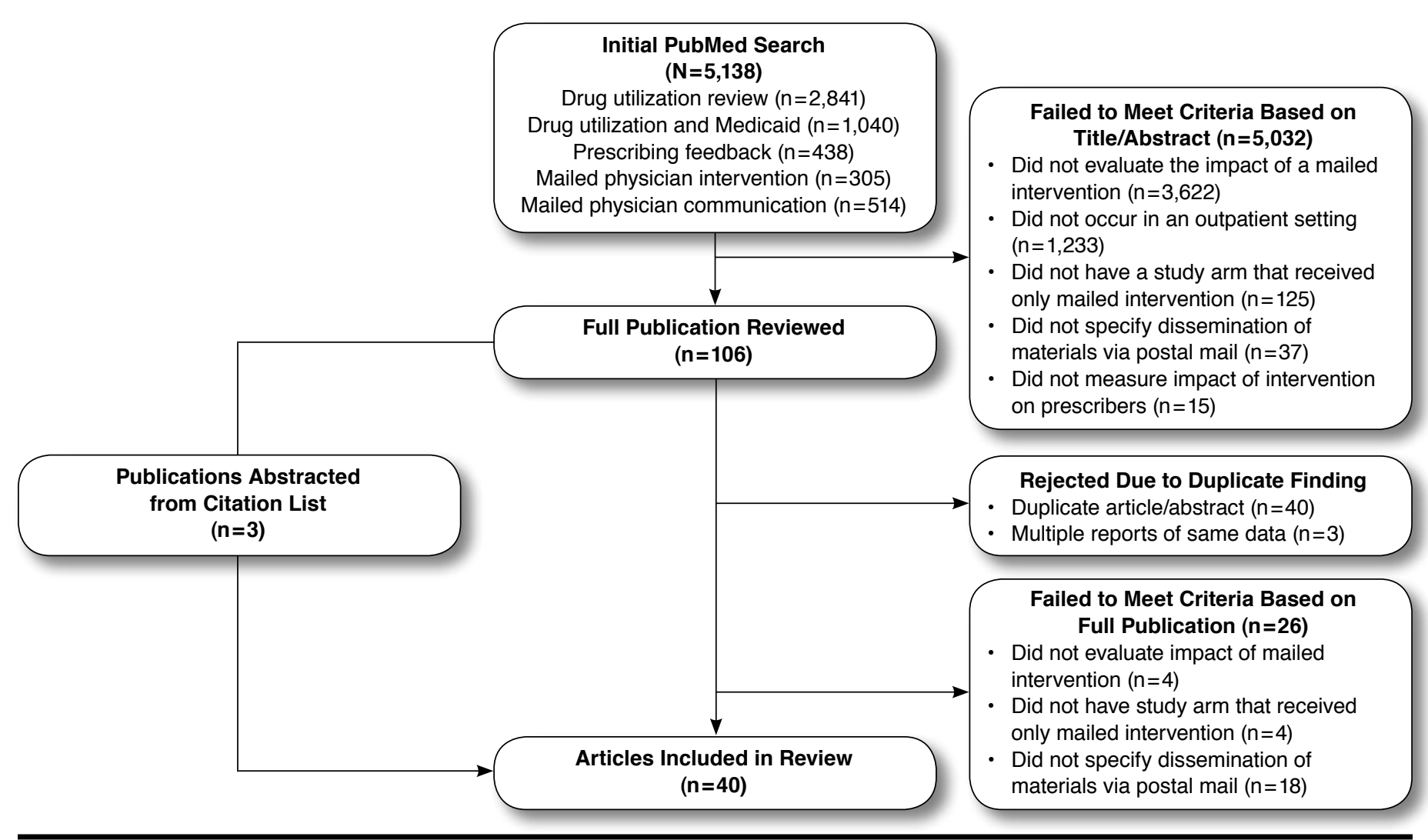

that were not identified during the electronic search. A similar review was performed for those identified reports.

Variables of assessment included follow-up period, data source, intervention targets, intervention materials, survey results of prescriber acceptance of the intervention provided, impact on prescribing patterns, impact on health care utilization, and impact on economic outcomes. All results were evaluated from the payer's perspective to determine the impact of direct correspondence-based programs. Throughout this review, the terms "significant" or "significance" was used to indicate a $P<0.05$.

\section{Results}

\section{Articles}

A series of 5 PubMed searches using the phrases "drug utilization review," "drug utilization review and Medicaid," "prescribing feedback," "mailed physician communication," and "mailed physician interventions" retrieved 5,138 articles. Of these, 106 articles were considered potentially relevant. Upon further review, several articles were excluded due to duplicate findings $(n=40)$ or multiple reports of the same data $(n=3)$. Several abstracts $(n=26)$ did not provide sufficient details to determine if the intervention met review inclusion criteria, requiring full publications to be reviewed. An additional 3 articles were identified by reviewing the reference lists of abstracted publications. The literature review yielded a total of 40 published reports that met review criteria.

\section{Study Characteristics}

Design, Setting, and Follow-Up Period. Table 1 displays the characteristics of each of the 40 studies included in this review. The majority of the programs used randomized controlled or quasi-experimental designs, and nearly half were conducted in U.S. state Medicaid agencies. Most programs allowed for a null period of 1 month or more to account for letter distribution and incorporation of recommendations. Programs that included letter distribution in the post-intervention follow-up period are noted in Table 1. Follow-up periods were generally $\leq 12$ months (36/39 [92.3\%]); only 3 programs used extended follow-up periods during which they reported quarterly or bi-yearly results as well as overall impact from baseline to completion. ${ }^{3,4,43}$

Audience. The majority of interventions were mailed to general 


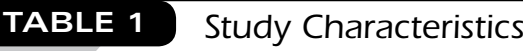

\begin{tabular}{|c|c|c|c|c|c|}
\hline Study & Study Design & Setting & $\begin{array}{l}\text { Follow-Up } \\
\text { Period } \\
\text { (Months) }\end{array}$ & Intervention Recipients & Data Sources \\
\hline Allard et al. (2001) ${ }^{47}$ & RCT & Quebec, Canada & $12^{\mathrm{a}}$ & Prescribers & $\begin{array}{l}\text { Survey responses from patient } \\
\text { identified via health care data- } \\
\text { base; prescription history as } \\
\text { recorded by home visit nurse; } \\
\text { health information obtained } \\
\text { from prescribers }\end{array}$ \\
\hline Anderson et al. (1996)26 & RCT & British Columbia, Canada & 6 & Prescribers $^{b}$ & $\begin{array}{l}\text { Triplicate Prescription Program } \\
\text { (monitors prescribing of narcot- } \\
\text { ics and certain anabolic steroids) }\end{array}$ \\
\hline $\begin{array}{l}\text { Avorn and Soumerai } \\
(1983)^{31}\end{array}$ & RCT & $\begin{array}{l}\text { Arkansas, New Hampshire, } \\
\text { Vermont, and District of } \\
\text { Columbia Medicaid }\end{array}$ & $9 c$ & Prescribers $^{b}$ & Claims database \\
\hline Bjornson et al (1990) 49 & RCT & Minnesota Medicaid & 4 & Prescribers & Claims database \\
\hline Coleman et al. (2003) 8,46 & $\mathrm{QE}$ & Connecticut Medicaid & 6 & Prescribers \& pharmacists & Claims database \\
\hline Collins et al. $(1997)^{13}$ & QE & Wisconsin Medicaid & 6 & Prescribers $\&$ pharmacists & Claims database \\
\hline Culbertson et al. (1999)14 & $\mathrm{QE}$ & Idaho Medicaid & 6 & Prescribers \& pharmacists & Claims database \\
\hline Dormuth et al. $(2004)^{44}$ & Clustered RCT & British Columbia, Canada & $3 \mathrm{~d}$ & Prescribers & Claims database \\
\hline Fick et al. $(2004)^{2}$ & $\begin{array}{l}\text { Randomized } \\
\text { block }\end{array}$ & U.S. Medicare & 6 & Prescribers & Claims database \\
\hline Groves $(1985)^{15}$ & Cross-sectional & Florida Medicaid & $3^{e}$ & Prescribers \& pharmacists & Claims database \\
\hline Guo et al. (1995) ${ }^{9}$ & $\mathrm{QE}$ & Alabama Medicaid & 7 & Prescribers & Claims database \\
\hline Hoffman et al. (2003)16 & $\begin{array}{l}\text { Retrospective } \\
\text { cohort }\end{array}$ & U.S. HMO & 6 & PCP & Claims database \\
\hline Hux et al. (1999)27 & RCT & Ontario, Canada & 6 & Prescribers ${ }^{\mathrm{b}}$ & Claims database \\
\hline Jing et al. $(2011)^{3}$ & QE & $\begin{array}{l}\text { Medicare and commercial } \\
\text { Health Plan of New York }\end{array}$ & $\begin{array}{c}\text { Physician arm: } \\
21^{\mathrm{f}} \\
\text { Patient arm: } \\
12^{\mathrm{f}}\end{array}$ & PCP \& patients & Claims database \\
\hline Kaufman et al. (2005)4 & $\mathrm{QE}$ & $\begin{array}{l}\text { Medicare and commercial } \\
\text { Health Plan of New York }\end{array}$ & $48^{\mathrm{f}}$ & $\begin{array}{l}\mathrm{PCP} \pm \text { prescriber if not the } \\
\text { same }\end{array}$ & Claims database \\
\hline Lee et al. (2004)10 & $\mathrm{QE}$ & Pennsylvania Medicaid & 9 & Prescribers & Claims database \\
\hline Meyer et al. (1991) ${ }^{17}$ & RCT & $\begin{array}{l}\text { Veterans Affairs Medical } \\
\text { Center in Colorado }\end{array}$ & 12 & $\begin{array}{l}\text { PCP identified as most fre- } \\
\text { quent prescriber }\end{array}$ & $\begin{array}{l}\text { Claims database and medical } \\
\text { records database }\end{array}$ \\
\hline Naughton et al. $(2007)^{32}$ & $\begin{array}{c}\text { Clustered } \\
\text { randomized trial }\end{array}$ & $\begin{array}{l}\text { Eastern Regional Health } \\
\text { Authority in Ireland }\end{array}$ & 6 & Prescribers & Claims database \\
\hline O'Connell et al. (1999)28 & Clustered RCT & Rural Australia & $9 g$ & Prescribers & Claims database \\
\hline Okano and Rascati (1995) ${ }^{18}$ & Clustered RCT & Texas Medicaid & 6 & Prescribers & Claims database \\
\hline \begin{tabular}{l|l} 
Owens et al. $(2008)^{50}$ \\
\end{tabular} & $\mathrm{QE}$ & Idaho Medicaid & 6 & Prescribers & Claims database \\
\hline Pimlott et al. $(2003)^{29}$ & RCT & Ontario, Canada & 6 & Prescribers ${ }^{b}$ & Claims database \\
\hline Raisch and Sleath (1999) ${ }^{19}$ & $\mathrm{QE}$ & New Mexico Medicaid & 3 & Prescribers & Claims database \\
\hline Rascati et al. (1996)20 & Clustered RCT & Texas Medicaid & 6 & Prescribers & Claims database \\
\hline Rokstad et al. $(1995)^{30}$ & QE & $\begin{array}{l}\text { Counties of More and } \\
\text { Romsdal, Norway }\end{array}$ & 1 & Prescribers & $\begin{array}{l}\text { Physician recorded report of } \\
\text { medications prescribed dur- } \\
\text { ing patient visits; pharmacy } \\
\text { recorded reports of medications } \\
\text { written on study-specific pre- } \\
\text { scription pads; administrative } \\
\text { recorded reports of physician } \\
\text { work hours }\end{array}$ \\
\hline Schectman et al. $(1995)^{45}$ & QE & Washington DC HMO & 6 & Prescribers & Claims database \\
\hline Seltzer et al. $(2000)^{21}$ & $\mathrm{QE}$ & Texas Medicaid & 10 & Prescribers & Claims database \\
\hline Shatin et al. $(2005)^{51}$ & $\begin{array}{l}\text { Time series } \\
\text { analysis }\end{array}$ & $\begin{array}{l}12 \text { health plans affiliated } \\
\text { with a U.S. managed } \\
\text { health company }\end{array}$ & 10 & Prescribers & Claims database \\
\hline Sleath and Collins (1997) ${ }^{48}$ & QE & New Mexico Medicaid & 3 & Prescribers & Claims database \\
\hline
\end{tabular}




\begin{tabular}{|c|c|c|c|c|c|}
\hline Study & Study Design & Setting & $\begin{array}{l}\text { Follow-Up } \\
\text { Period } \\
\text { (Months) }\end{array}$ & Intervention Recipients & Data Sources \\
\hline Sleath et al. $(1997)^{22}$ & $\mathrm{QE}$ & New Mexico Medicaid & 12 & Prescribers \& pharmacists & Claims database \\
\hline Smith et al. (1997) 23 & Clustered RCT & Washington Medicaid & 3 & $\begin{array}{c}\text { Prescribers or pharmacists } \\
\text { if prescriber information } \\
\text { was not available }\end{array}$ & Claims database \\
\hline Søndergaard et al. $(2002)^{6}$ & RCT & County of Funen, Denmark & 12 & Prescribers & $\begin{array}{l}\text { Pharmacoepidemiologic and } \\
\text { billing database }\end{array}$ \\
\hline Søndergaard et al. $(2003)^{5}$ & RCT & County of Funen, Denmark & 12 & Prescribers & $\begin{array}{l}\text { Pharmacoepidemiologic and } \\
\text { billing database }\end{array}$ \\
\hline Starner et al. (2009) ${ }^{24}$ & QE & $\begin{array}{c}\text { Illinois, New Mexico, } \\
\text { Oklahoma, Texas Medicare }\end{array}$ & 1 day & Prescribers & Claims database \\
\hline Vaegter et al. (2010)43 & Time series analysis & Størstrom County, Denmark & $84 \mathrm{~h}$ & Prescribers & Claims database \\
\hline Woodward et al (2008)7 & QE & $\begin{array}{l}\text { Australian Department of } \\
\text { Veterans Affairs }\end{array}$ & $\begin{array}{l}\text { Polypharmacy } \\
\text { arm: } 3 \\
\text { Drug interac- } \\
\text { tion arm: } \\
45 \text { days } \\
\end{array}$ & $\begin{array}{l}\text { PCP identified as most } \\
\text { frequent prescriber }\end{array}$ & Claims database \\
\hline Zimmerman et al. (1994) 11 & QE & Wisconsin Medicaid & 12 & Prescribers & Claims database \\
\hline Zuckerman et al. (2004)25 & QE & Pennsylvania Medicaid & 5 & Prescribers & $\begin{array}{l}\text { Claims database, meta-analysis } \\
\text { by Yusuf et al. (1985) }{ }^{54} \text { used to } \\
\text { estimate event probabilities for } \\
\text { economic analysis }\end{array}$ \\
\hline Zuckerman et al. (2004)12 & $\mathrm{QE}$ & Pennsylvania Medicaid & 6 & Prescribers & Claims database \\
\hline 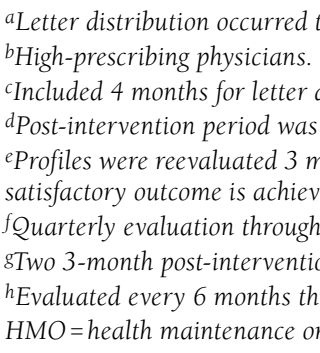 & $\begin{array}{l}\text { throughout entire 12-mo } \\
\text { distribution. } \\
8 \text { weeks for one 2-part } \\
\text { nonths after intervention } \\
\text { ed. } \\
\text { tout entire post-intervent } \\
\text { on follow-up periods me } \\
\text { roughout entire post-inte } \\
\text { rganization; } P C P=\text { prime }\end{array}$ & $\begin{array}{l}\text { onth post-intervention follow-up } \\
\text { mailing that was treated as a si } \\
\text { n mailing. If an acceptable presc } \\
\text { ation follow-up period. } \\
\text { easured } 3 \text { months apart. } \\
\text { tervention follow-up period. } \\
\text { hary care physicians; } Q E=q u a s i-\end{array}$ & $\begin{array}{l}\text { period. } \\
\text { ingle interventior } \\
\text { criber response o }\end{array}$ & hange in therapy has not occur & red, the case is followed until a \\
\hline
\end{tabular}

physicians rather than specialists; few interventions specified inclusion of prescribers to include midlevel practitioners, such as nurse practitioners or physician assistants, as permitted by the practice law in the area. Interventions were most commonly sent exclusively to prescribers involved in the care of a patient who had received a target medication (32/39 [82.1\%]). Five programs included the primary care provider as an intervention recipient regardless if they had prescribed the target medication, $3,4,7,16,17$ and 4 targeted those identified as "high-prescribers" based on a program-specified standard or in comparison to peers. ${ }^{26,27,29,31}$ Hux et al. (1999) $)^{27}$ and Pimlott et al. $(2003)^{29}$ selected prescribers who had written $\geq 10$ prescriptions for a target medication in a 2-month period, while Anderson et al. $(1996)^{26}$ selected those who had written excessively for target agents relative to the overall prescribing population. Programs $(n=8)$ that selected prescribers based on geographic location rather than prescribing behavior were found to have little to no impact on prescribing patterns. $5,6,28,30,32,43-45$

In addition to targeting prescribers, several programs $(n=6)$ also sent correspondence to pharmacies involved in the care of identified patients. ${ }^{8,13-15,22,23,46}$ Jing et al. (2011) was the only identified program that included both prescribers and patients as intervention recipients. ${ }^{3}$ Six of the 7 aforementioned programs concluded that inclusion of a second recipient to be more effective at achieving the targeted outcome as compared with prescriber recipients alone. Culbertson et al. (1999) was the only program to note no difference in effect when the same intervention material was sent to physicians and pharmacists as compared with physicians alone. ${ }^{14}$

Data Source. Despite known limitations, the majority of identified programs used prescription and medical claims databases to obtain medication use and diagnostic histories. Groves (1985) used a combination of computerized alert tools to identify patients with potentially inappropriate medications 
followed by an audit by clinical pharmacists. ${ }^{15}$ Two (5.1\%) programs utilized patient- or practitioner-reported information as a primary data source. After identifying patients from a government-supported health care database, Allard et al. (2001) utilized patient- and prescriber-reported medication and health information. ${ }^{47}$ Rokstad et al. (1995) obtained information through physician recordings of medications prescribed, pharmacy reportings of medications prescribed on study-specific prescription pads, and physician staff recordings of physician work hours. ${ }^{30}$ The use of multiple strategies may improve patient identification but did not always translate to improved prescribing patterns.

\section{Intervention Strategies}

Most programs (34/39 [87.2\%]) evaluated change as compared with pre-intervention prescribing behavior or in comparison with a control group who received no intervention materials. Of these, 14 were randomized controlled trials. Ten programs compared changes in prescribing behavior between multiple intervention strategies. Fick et al. (2004) demonstrated that providing patient-identifying intervention material along with a list of alternative medications was more effective than mailed educational material alone. ${ }^{2}$ Meyer et al. (1991) found no difference among a patient-identifying intervention that provided general or specific recommendations. ${ }^{17}$ Both studies took precautions to avoid cross-contamination of the intervention groups. Additionally, no difference was found among interventions that provided various forms of nonpatient-identifying information, such as educational material or prescriber feedback. ${ }^{5,6,45}$ Combining nonpatient-identifying intervention materials with academic detailing produced mixed results. ${ }^{26,31,32}$ Table 2 describes the association between mailed intervention and change in prescribing as reported in each study included in this review.

Target Medications. Focusing an intervention on specific medications appears to be a successful means of impacting prescribing behavior. Okano and Rascati $(1995)^{18}$ and Raisch and Sleath $(1999)^{19}$ used similar intervention techniques to address inappropriate prescribing of antiulcer agents. Okano and Rascati focused the intervention on a single target and successfully reduced the use of dual antiulcer therapy. ${ }^{18}$ When Raisch and Sleath expanded target criteria to include long-term and high-dose therapies without an appropriate diagnosis, no impact was seen on the use of dual antiulcer therapy. ${ }^{19}$ Three programs that targeted an extensive list of unrelated medications and/or disease states had minimal impact on prescribing behavior. ${ }^{28,43,44}$ The exception to using broad targets is Groves, who successfully used patient-specific recommendations to evoke change in prescribing among patients identified as atrisk for drug-related adverse effects. ${ }^{15}$

Several authors noted that it may be difficult to prompt discontinuation of agents that have been used on a long-term basis; this may be particularly true for sedative hypnotics. $13,24,29,48$ One report found that patient demand deterred 26\% of prescribers from intervening on long-term use of sedative agents. ${ }^{48}$ Additionally, both prescribers and patients may have a decreased perception of potential harm associated with agents that have been used on a long-term basis. ${ }^{29}$ Aside from the potential impact of chronic use, there appeared to be no difference in success rates between interventions that recommended initiation or discontinuation of a medication.

Intervention Materials. Provision of patient-identifying information appears to be a major factor contributing to the success of an intervention. Patient-identifying material ranged from simply identifying a patient by name to providing detailed prescription and medical claims histories or medication adherence reports. Twenty-one of the 25 programs that provided some form of patient-identifying data were considered to have a significant impact on prescribing. . $-4,7-16,18-23,25,48$ It should be noted that Meyer et al. considered their patient-identifying intervention to be unsuccessful because changes in prescribing patterns were no longer significant at 6 and 12 months postintervention. ${ }^{17}$ Though Bjornson et al. (1990) provided patientidentifying data, the data differed from each of the successful interventions-instead of providing information regarding all eligible patients, they included a medication history profile for one patient under each physician's care and monitored for prescribing changes within that single identified profile. ${ }^{49}$

Most programs that used patient-identifying data opted to make generalized recommendations that could be applicable to their entire target population. ${ }^{2-4,7-14,16,18-25,48,49}$ For example, the intervention conducted by Zuckerman et al. (2004) alerted physicians of the identified patient's potential for acetaminophen overuse, discussed the associated risks, and suggested adjusting therapy to meet recommended daily standards. ${ }^{12}$ Such a method appears to be an effective intervention tactic, as 20 of 22 programs (90.1\%) that provided this type of recommendation were successful. Programs that provided exclusively patient-specific recommendations often enlisted a team of physicians and pharmacists who carefully reviewed profiles and developed customized therapeutic suggestions..$^{15,47}$ Kaufman et al. (2005) was unique in that they provided a telephone followup to the subset of physicians identified as the highest volume prescribers during which they offered patient specific recommendations. ${ }^{4}$ Only 1 program sought to compare the impact of such interventions and found no difference between generalized versus patient-specific recommendations. ${ }^{17}$

Several programs measured the impact of incorporating academic outreach into a mailed intervention., ${ }^{4,26,31,32}$ In this review, all direct, nonmailed communications were classified as academic outreach and included live group education, individual visits from academic detailers, and telephone 


\section{TABLE 2 Association Between Mailed Intervention, Prescribing Patterns, and Health Care Utilization}

\begin{tabular}{|c|c|c|c|}
\hline Study & Target Medications & $\begin{array}{l}\text { Program Description } \\
\text { and Intervention Materials }\end{array}$ & $\begin{array}{l}\text { Effects of Mailed Intervention } \\
\text { on Prescribing Behavior }\end{array}$ \\
\hline Allard et al. (2001) ${ }^{47}$ & $\begin{array}{l}\text { PIP per Quebec } \\
\text { Committee on } \\
\text { Drug Use in the } \\
\text { Elderly } 55\end{array}$ & $\begin{array}{l}\text { Patients }>75 \text { years with }>3 \text { unique medications } \\
\text { divided into intervention }(\mathrm{n}=136) \text { and control } \\
(\mathrm{n}=130) \text {. } \\
\text { Intervention patient profiles reviewed by team } \\
\text { of } 2 \text { physicians, } 1 \text { pharmacist, and } 1 \text { nurse. } \\
\text { Recommendations and supporting references } \\
\text { were mailed to prescribers on behalf of } 80 \\
\text { patients. }\end{array}$ & $\begin{array}{l}\text { Mean number [SD] of PIP per patient decreased by } \\
0.24[0.69] \text { in intervention and } 0.15[0.52] \text { in control } \\
(P=\text { NS as compared with baseline and }<0.001 \text { as com- } \\
\text { pared with control). } \\
\text { Number of patients with } \geq 1 \text { PIP decreased } 18(14.2 \%) \text { in } \\
\text { intervention and } 8(6.9) \text { in control }(P=\mathrm{NS} \text { as compared } \\
\text { with baseline and } 0.049 \text { as compared with control). } \\
\text { No difference in number of different medications or } \\
\text { global assessment of medication use between groups. }\end{array}$ \\
\hline Anderson et al. (1996)26 & $\begin{array}{l}\text { Narcotics and other } \\
\text { analgesics, narcotic } \\
\text { antitussives, certain } \\
\text { anabolic steroids }\end{array}$ & $\begin{array}{l}\text { Physicians }(n=18) \text { received mailed notification } \\
\text { that they had been identified as a high pre- } \\
\text { scriber of regulated analgesics relative to peers. } \\
\text { Physicians }(n=18) \text { received mailed notification } \\
\text { and attended an educational workshop. } \\
\text { Physicians }(n=18) \text { served as a control group. }\end{array}$ & $\begin{array}{l}\text { Mean number [SD] of prescriptions issued per phy- } \\
\text { sician decreased by } 29.2 \text { [32.1] ( } 25 \% \text { reduction) in } \\
\text { mailed intervention alone and by } 33.4 \text { [37.3] ( } 33 \% \\
\text { reduction) in mailed intervention + education. ( } P<0.01 \\
\text { for both as compared to control and NS between } \\
\text { groups). } \\
3.8(3 \%) \text { increase in number of prescriptions issued } \\
\text { observed in control. }\end{array}$ \\
\hline $\begin{array}{l}\text { Avorn and Soumerai } \\
(1983)^{31}\end{array}$ & $\begin{array}{l}\text { Cerebral and } \\
\text { peripheral vasodi- } \\
\text { lators, cephalexin, } \\
\text { propoxyphene }\end{array}$ & $\begin{array}{l}\text { Physicians ( } \mathrm{n}=132) \text { received a cover letter fol- } \\
\text { lowed by a series of } 3 \text { educational bulletins } \\
\text { without literary or visual appeal alone }(\mathrm{n}=66) \\
\text { or in addition to a series of } 6 \text { visually and liter- } \\
\text { ary appealing educational "unadvertisements" } \\
\text { and supplemental patient education material } \\
(\mathrm{n}=66) \text {. } \\
\text { Physicians ( } \mathrm{n}=141) \text { received academic detailing } \\
\text { in addition to all print materials. } \\
\text { Physicians }(\mathrm{n}=140) \text { served as a control group. }\end{array}$ & $\begin{array}{l}\text { Mailed only prescribed an average of } 251 \text { fewer units } \\
\text { of target drugs as compared with control }(P=\mathrm{NS}) \text { and } \\
\text { mailed + academic detailing prescribed an average of } \\
782 \text { fewer units as compared with control }(P<0.001) \text {. } \\
\text { No differences observed for various forms of mailed } \\
\text { materials alone. }\end{array}$ \\
\hline Bjornson et al. (1990)49 & $\begin{array}{l}\text { Hydralazine, iso- } \\
\text { sorbid dinitrate, } \\
\text { prazosin }\end{array}$ & $\begin{array}{l}\text { Physicians }(\mathrm{n}=288) \text { received a cover letter, } \\
\text { published clinical trial on pharmacologic man- } \\
\text { agement of congestive heart failure, a patient } \\
\text { medication history profile, and response form. } \\
\text { Physicians ( } \mathrm{n}=288 \text { ) served as a control group. }\end{array}$ & $\begin{array}{l}\text { In both groups, } 5(0.9 \%) \text { physicians prescribed a full } \\
\text { change in therapy for the identified patients, and } 23 \\
(4 \%) \text { prescribed a partial change in therapy for the } \\
\text { identified patients ( } P=\text { NS for both and combined } \\
\text { effect). }\end{array}$ \\
\hline$\overline{\text { Coleman et al. }(2003)^{8}}$ & $\begin{array}{l}\text { SABA, inhaled } \\
\text { corticosteroids, leu- } \\
\text { kotriene modifiers, } \\
\text { mast-cell stabiliz- } \\
\text { ers, oral cortico- } \\
\text { steroids, long- } \\
\text { acting beta2- } \\
\text { agonists }\end{array}$ & $\begin{array}{l}\text { Prescribers and pharmacies of patients }(\mathrm{n}=35) \\
\text { identified as high dose SABA users received a } \\
\text { cover letter, patient medication profile, and an } \\
\text { educational insert. } \\
\text { Patients identified as normal dose SABA users } \\
(\mathrm{n}=510) \text { served as a comparison group. }\end{array}$ & $\begin{array}{l}46 \% \text { of patients in the intervention group were no lon- } \\
\text { ger considered high-dose SABA users }(P<0.001) \text {. } \\
\text { Prior to the intervention, fewer intervention patients } \\
\text { received } \geq 1 \text { long-term controller agent }(78[58 \%] \text { vs. } \\
491[96 \%] \text { for intervention and comparison, respec- } \\
\text { tively; } P<0.001) \text {; no difference between groups follow- } \\
\text { ing intervention ( } 87 \text { [65\%] vs. } 360 \text { [71\%], respectively; } \\
P=\text { NS). } \\
\text { No pre-post difference in intervention for any target } \\
\text { long-term controller agent ( } P=\text { NS). } \\
\text { Prior to intervention, more patients in the intervention } \\
\text { group had claims for office visits }(P<0.001) \text {. No differ- } \\
\text { ence between groups following intervention }(P=N S) \\
\text { and a within-group decreases in both intervention and } \\
\text { comparison groups ( } P<0.05) \text {. } \\
\text { Prior to the intervention, more patients in the com- } \\
\text { parison group had claims for physician services } \\
(P<0.001) \text {. No difference between groups following } \\
\text { intervention }(P=N S) \text { within-group decrease in com- } \\
\text { parison group }(P<0.05) \text {. } \\
\text { No between or within group differences for hospital } \\
\text { visits, emergency department visits, or hospitalized } \\
\text { days. }\end{array}$ \\
\hline
\end{tabular}




\begin{tabular}{|c|c|c|c|}
\hline Study & Target Medications & $\begin{array}{c}\text { Program Description } \\
\text { and Intervention Materials }\end{array}$ & $\begin{array}{c}\text { Effects of Mailed Intervention } \\
\text { on Prescribing Behavior }\end{array}$ \\
\hline Collins et al. (1997)13 & $\begin{array}{l}\text { Dipyridamole } \\
\text { and related agents } \\
\text { (defined as aspirin, } \\
\text { sulfinpyrazone, } \\
\text { and ticlopidine) }\end{array}$ & $\begin{array}{l}\text { Intervention packet contained letter identify- } \\
\text { ing potential dipyridamole problem, refer- } \\
\text { ence information on dipyridamole use, list of } \\
\text { patient names seen by the physician and/or } \\
\text { pharmacist, and patient dipyridamole drug use } \\
\text { histories. } \\
\text { Physicians alone }(n=203) \text { received intervention } \\
\text { for long-term care }(n=181) \text { and ambulatory } \\
(n=99) \text { patients. } \\
\text { Pharmacists alone }(n=157) \text { received interven- } \\
\text { tion for long-term care }(n=149) \text { and ambula- } \\
\text { tory }(n=132) \text { patients. } \\
\text { Physicians (n=182) + pharmacists (n=147) } \\
\text { received intervention for long-term care }(n=91) \\
\text { and ambulatory }(n=120) \text { patients. } \\
\text { Long-term care }(n=342) \text { and ambulatory } \\
\text { (n=235) patients served as a control group. }\end{array}$ & $\begin{array}{l}42(46.2 \%) \text { long-term care (OR: } 2.10 ; P<0.025) \text { and } \\
47(39.2 \%) \text { ambulatory patients (OR: } 3.81 ; P<0.025) \\
\text { in the physician-pharmacist group had no claim for } \\
\text { dipyridamole. } \\
57(31.5 \%) \text { long-term care (OR: } 1.31 ; P=N S) \text { and } 30 \\
(30.3 \%) \text { ambulatory patients (OR: } 2.22 ; P<0.025) \text { in } \\
\text { physician-only group had no claim for dipyridamole. } \\
37(24.8 \%) \text { long-term care (OR: } 0.92) \text { and } 30(22.7 \%) \\
\text { ambulatory patients (OR: } 1.67) \text { in pharmacist only } \\
\text { group had no claim for dipyridamole }(P=\text { NS for both). } \\
87 \text { ( } 25.4 \%) \text { long-term care and } 36(15.3 \%) \text { ambulatory } \\
\text { patients in control group had no claim for dipyridamole. }\end{array}$ \\
\hline Culbertson et al. (1999)14 & $\begin{array}{l}\text { Cimetidine, famoti- } \\
\text { dine, lansoprazole, } \\
\text { nizatidine, omepra- } \\
\text { zole, ranitidine, } \\
\text { sucralfate }\end{array}$ & $\begin{array}{l}\text { Intervention packets which contained a cover } \\
\text { letter, educational material, medication pro- } \\
\text { files of patient identified as receiving poten- } \\
\text { tially inappropriate antiulcer therapy, and a } \\
\text { response form were sent to physicians and/or } \\
\text { pharmacies. } \\
\text { Physicians ( } n=113) \text { received intervention } \\
\text { regarding } 138 \text { patients. } \\
\text { Physicians }(n=187)+\text { pharmacies }(n=79) \\
\text { received intervention regarding } 329 \text { patients. } \\
\text { Physicians ( } n=158)+ \text { pharmacies ( } n=60) \\
\text { received intervention and follow-up calls } \\
\text { regarding } 248 \text { patients. }\end{array}$ & $\begin{array}{l}12.4 \% \text { decrease in dose }{ }^{a} \text { in physician alone, } 8.0 \% \\
\text { decrease in physicians }+ \text { pharmacists, } 14.0 \% \text { decrease } \\
\text { in physicians }+ \text { pharmacists }+ \text { follow-up call: } 14.0 \% \\
\text { decrease }(P<0.01 \text { for all). } \\
\text { Percent change greater in physician + pharmacist + } \\
\text { follow-up call as compared with physician + pharma- } \\
\text { cist }(P<0.05) \text { but not physician alone }(P=N S) \text {. }\end{array}$ \\
\hline Dormuth et al. (2004) 44 & $\begin{array}{l}\text { Cimetidine, metro- } \\
\text { nidazole/(amoxicil- } \\
\text { lin or tetracycline), } \\
\text { aspirin/ibuprofen/ } \\
\text { naproxen, iso- } \\
\text { sorbide dinitrate, } \\
\text { thiazide diuretics, } \\
\text { inhaled cortico- } \\
\text { steroids, CCB, } \\
\text { long-acting BDZ, } \\
\text { hormones, clonaz- } \\
\text { epam/alprazolam, } \\
\text { diazepam, finas- } \\
\text { teride }\end{array}$ & $\begin{array}{l}\text { Physicians }(\mathrm{n}=258) \text { received a series of edu- } \\
\text { cational bulletins that provided a clear recom- } \\
\text { mendation for prescribing each target medica- } \\
\text { tion or class. } \\
\text { Physicians }(\mathrm{n}=241) \text { received the educational } \\
\text { bulletin } 3-8 \text { months later with the exception } \\
\text { of a } 2 \text {-part series that was mailed after } 8 \text { weeks } \\
\text { and served as a control group. }\end{array}$ & $\begin{array}{l}\text { Trends in prescribing of target medication to newly } \\
\text { treated patients proceeded in direction suggested by } \\
\text { mailing } 1.3 \text { times more likely in intervention group } \\
\text { (95\% CI 1.13-1.52). } \\
\text { No single letter was associated with a significant } \\
\text { impact on prescribing behavior. }\end{array}$ \\
\hline Fick et al. $(2004)^{2}$ & $\begin{array}{l}1997 \text { Beers crite- } \\
\text { ria list of agents } \\
\text { to avoid in older } \\
\text { adults }^{56}\end{array}$ & $\begin{array}{l}\text { All in-network PCPs }(n=355) \text { received mailed } \\
\text { educational material and a copy of the } 1997 \\
\text { Beers criteria. } 56 \\
3 \text { months later, physicians }(n=170) \text { received a } \\
\text { personally addressed letter listing patients }>65 \\
\text { years who received } \geq 1 \text { PIP as well as a detailed } \\
\text { educational brochure. Intervention packets } \\
\text { were sent quarterly for } 12 \text { months. Physicians } \\
\text { who had not written a PIP received acknowl- } \\
\text { edgement. } \\
\text { Physicians }(n=185) \text { served as a control group. }\end{array}$ & $\begin{array}{l}\text { Number of patients who received } \geq 1 \text { PIP decreased } \\
\text { from } 3,364(19.4 \%) \text { at baseline to } 3,007(17.9 \%) \\
(P<0.001) \text {. }\end{array}$ \\
\hline
\end{tabular}




\begin{tabular}{|c|c|c|c|}
\hline Study & Target Medications & $\begin{array}{l}\text { Program Description } \\
\text { and Intervention Materials }\end{array}$ & $\begin{array}{l}\text { Effects of Mailed Intervention } \\
\text { on Prescribing Behavior }\end{array}$ \\
\hline Groves $(1985)^{15}$ & $\begin{array}{l}\text { NSAIDS, tricyclic } \\
\text { antidepressants, } \\
\text { antipsychotic } \\
\text { tranquilizers, } \\
\text { antihypertensive } \\
\text { and diuretics, } \\
\text { antidiabetic agents, } \\
\text { cardiac agents }\end{array}$ & $\begin{array}{l}\text { Computer-generated medical history profiles } \\
\text { identified patients at risk for drug interactions, } \\
\text { adverse reactions, and potential overuse/under- } \\
\text { use of medications. A team of } 4 \text { pharmacists } \\
\text { and } 1 \text { physician conducted monthly reviews of } \\
\text { identified profiles to determine if a interven- } \\
\text { tion was necessary. } \\
\text { If warranted, a patient-specific letter describ- } \\
\text { ing the problem was mailed to the prescribing } \\
\text { physician, diagnosing physician, and dispens- } \\
\text { ing pharmacist. } \\
\text { The review committee followed up on each } \\
\text { case until a satisfactory outcome was reached. }\end{array}$ & $\begin{array}{l}\text { After } 9 \text { months, } 443 \text { (54\%) of cases were considered } \\
\text { resolved (as defined by a change in therapy or receipt } \\
\text { of an acceptable prescriber explanation for continua- } \\
\text { tion in therapy). }\end{array}$ \\
\hline Guo et al. (1995)9 & $\begin{array}{l}\text { Nizatidine, sucral- } \\
\text { fate, famotidine, } \\
\text { omeprazole, raniti- } \\
\text { dine, cimetidine }\end{array}$ & $\begin{array}{l}\text { Prescribers }(n=118) \text { were sent a cover letter, } \\
\text { profile of patient identified as receiving poten- } \\
\text { tially inappropriate antiulcer therapy, and a } \\
\text { comment form. } \\
\text { Prescribers }(n=3776) \text { who did not prescribe } \\
\text { potentially inappropriate antiulcer therapy } \\
\text { served as a comparison group. }\end{array}$ & $\begin{array}{l}\text { Significant downward trend in days of therapy }{ }^{\mathrm{b}} \\
\text { throughout follow-up period }(P=0.038) \text {. } \\
\text { Decrease in days of therapy }{ }^{\mathrm{b}} \text { significant in fifth month } \\
\text { of } 8 \text {-month follow-up period }(P<0.05) \text {. No significant } \\
\text { changes noted in other individual months. }\end{array}$ \\
\hline Hoffman et al. (2003)16 & $\begin{array}{l}\text { Medications with } \\
\text { high-abuse poten- } \\
\text { tial (not specified) }\end{array}$ & $\begin{array}{l}\text { PCPs of patients }(n=94) \text { that triggered } \geq 3 \\
\text { categories of abuse potential alerts within } 1 \\
\text { calendar quarter received a prescription pro- } \\
\text { file for each identified patient and a letter that } \\
\text { recommended reevaluation of the patient's } \\
\text { medication use. } \\
\text { Patients }(n=89) \text { triggering } 1-2 \text { abuse potential } \\
\text { alerts within } 1 \text { calendar quarter served as a } \\
\text { matched comparison group. }\end{array}$ & $\begin{array}{l}\text { Mean number }[S D] \text { of high-abuse prescription claims } \\
\text { per patient per month decreased by } 3.06[4.61] \text { in } \\
\text { intervention and } 1.96[2.64] \text { in comparison }(P<0.05) \text {. } \\
\text { Mean number }[S D] \text { of prescribers per patient } \\
\text { decreased by } 2.2[3.26] \text { in intervention and } 1.36[2.43] \\
\text { in comparison }(P<0.05) \text {. } \\
\text { Mean number }[S D] \text { of prescription claims per member } \\
\text { per month decreased by } 4.97[4.43] \text { in intervention } \\
\text { and } 3.72[4.68] \text { in comparison }(P=N S) \text {. }\end{array}$ \\
\hline Hux et al. (1999)27 & $\begin{array}{l}\text { Pencillins, } \\
\text { macrolides, } \\
\text { trimethoprim- } \\
\text { sulfamethaxazole, } \\
\text { tetracycline, first- } \\
\text { generation cephalo- } \\
\text { sporins, ciprofloxa- } \\
\text { cin, norfloxacin, } \\
\text { cefaclor, others (not } \\
\text { specified) }\end{array}$ & $\begin{array}{l}\text { Physicians }(\mathrm{n}=134) \text { received prescriber feed- } \\
\text { back displaying individual and peer antibiotic } \\
\text { prescribing patterns as well as guideline-based } \\
\text { educational materials every } 2 \text { months for } 6 \\
\text { months. } \\
\text { Physicians }(\mathrm{n}=116) \text { received prescribing pro- } \\
\text { files on } 1 \text { occasion after the completion of } \\
\text { feedback in the intervention and served as a } \\
\text { control group. }\end{array}$ & $\begin{array}{l}\text { Proportion of first-line antibiotics use increased by } \\
2.6 \% \text { in intervention and decreased by } 1.7 \% \text { in control } \\
(P<0.01) \text {. }\end{array}$ \\
\hline Jing et al. $(2011)^{3}$ & $\begin{array}{l}\text { Miscellaneous } \\
\text { antidiabetic and } \\
\text { antihypertensive } \\
\text { agents }\end{array}$ & $\begin{array}{l}\text { Quarterly letters and medication profiles } \\
\text { were sent to PCPs of potentially nonadherent } \\
\text { patients. } \\
\text { After } 4 \text { quarters, supplemental educational } \\
\text { material was also mailed to patients. }\end{array}$ & $\begin{array}{l}\text { Prescribers alone: number of potentially nonadherent } \\
\text { patients decreased from 10,722 }(35.6 \%) \text { at baseline to } \\
9,701(30.8 \%) \text { over } 3 \text { calendar quarters }(P<0.001) \text {. } \\
\text { Prescribers + patients: number of potentially non- } \\
\text { adherent patients decreased from } 9,701(30.8 \%) \text { at } \\
\text { baseline to } 9,086(27.7 \%) \text { over } 4 \text { calendar quarters } \\
(P<0.001) \text {. }\end{array}$ \\
\hline Kaufman et al. (2005) & $\begin{array}{l}\text { Quarters 1-9: ami- } \\
\text { triptyline, cyclo- } \\
\text { benzaprine, diaz- } \\
\text { epam, indometha- } \\
\text { cin, meprobamate, } \\
\text { methocarbamol, } \\
\text { methyldopa, pro- } \\
\text { poxyphene } \\
\text { Quarters 10-16: } \\
\text { amitriptyline, } \\
\text { cyclobenzaprine, } \\
\text { diazepam, dicy- } \\
\text { clomine, disopyra- } \\
\text { mide, hyoscyamine, } \\
\text { indomethacin, } \\
\text { meprobamate, } \\
\text { methocarbamol, } \\
\text { methyldopa }\end{array}$ & $\begin{array}{l}\text { Physicians received quarterly intervention pack- } \\
\text { ets that contained a cover letter, list of patients } \\
>65 \text { years who received a PIP, name of PIP } \\
\text { received, and a list of formulary alternatives. } \\
\text { Each quarter, a clinical pharmacist contacted } \\
\text { the highest volume prescribers via telephone to } \\
\text { offer recommendations. } \\
\text { All in-network providers and pharmacists } \\
\text { received mailed educational information. }\end{array}$ & $\begin{array}{l}\text { Number of patients who filled } \geq 1 \text { PIP decreased from } \\
2,871(5.3 \%) \text { to } 1,451(2.2 \%)(P<0.001) \text {. } \\
\text { Number of patients who received } \geq 1 \text { of the } 7 \text { agents } \\
\text { that were targets throughout the entire intervention } \\
\text { decreased from } 1,398(2.6 \%) \text { to } 390(0.6 \%)(P<0.001) \text {. }\end{array}$ \\
\hline
\end{tabular}




\begin{tabular}{|c|c|c|c|}
\hline Study & Target Medications & $\begin{array}{l}\text { Program Description } \\
\text { and Intervention Materials }\end{array}$ & $\begin{array}{l}\text { Effects of Mailed Intervention } \\
\text { on Prescribing Behavior }\end{array}$ \\
\hline Lee et al. (2004)10 & SABA, salmeterol & $\begin{array}{l}\text { Physicians }(n=564) \text { received a list of pediatric } \\
(5-18 \text { years }) \text { patients with potentially inap- } \\
\text { propriate SABA }(n=382) \text { or salmeterol }(n=35) \\
\text { use, patient-specific prescription and medical } \\
\text { claims data, and a patient diary. } \\
3 \text { months post-intervention, a monograph that } \\
\text { provided continuing medical education credits } \\
\text { was sent to all physicians likely to treat pedi- } \\
\text { atric asthma. }\end{array}$ & $\begin{array}{l}\text { Among high-dose SABA users, use of SABA metered- } \\
\text { dose inhaler decreased by } 26.3 \%(P<0.001) \text {; no change } \\
\text { among other dosage forms }(P=N S) \text {. Use of mast-cell } \\
\text { stabilizer metered-dose inhaler decreased by } 36.9 \% \\
\text { and use of leukotriene modifiers increased by } 36.6 \% \\
(P<0.05 \text { for all); no change among asthma controller } \\
\text { agents. No change in emergency department visits or } \\
\text { hospitalizations from baseline ( } P=\text { NS). } \\
18 \text { ( } 82 \%) \text { of salmeterol-only recipients discontinued } \\
\text { use or received a SABA. }\end{array}$ \\
\hline Meyer et al. (1991)17 & $\begin{array}{l}\text { All medications } \\
\text { excluding topical, } \\
\text { ocular, and medical } \\
\text { supplies }\end{array}$ & $\begin{array}{l}\text { Prescribers received simple intervention on } \\
\text { behalf of patients }(\mathrm{n}=102) \text { with } \geq 10 \text { active } \\
\text { prescriptions that consisted of a list of identi- } \\
\text { fied patients and a request to reduce number of } \\
\text { medications. } \\
\text { Prescribers received an intensive intervention } \\
\text { on behalf of patients }(\mathrm{n}=104) \text { that consisted of } \\
\text { simple intervention material plus patient-spe- } \\
\text { cific recommendations for reducing polyphar- } \\
\text { macy and an estimate of patient's compliance } \\
\text { with the drug regimen. } \\
\text { Patients }(n=88) \text { served as a control group. }\end{array}$ & $\begin{array}{l}\text { The number of medications was reduced in all groups } \\
\text { at } 4,6 \text {, and } 12 \text { months in all groups }(P=0.001) \text {. } \\
\text { The combined effect of the two intervention was } \\
\text { significant at } 4 \text { months }(P=0.03) \text {, but not at } 6 \text { or } 12 \\
\text { months ( } P>\text { NS). } \\
\text { No difference was observed between the simple and } \\
\text { intensive intervention ( } P=\text { NS). }\end{array}$ \\
\hline Naughton et al. (2007)32 & $\begin{array}{l}\text { Antiplatelet agents, } \\
\text { HMG-CoA reduc- } \\
\text { tase inhibitors }\end{array}$ & $\begin{array}{l}\text { Prescribers }(\mathrm{n}=50) \text { received feedback display- } \\
\text { ing number of CVD and diabetic patients not } \\
\text { receiving recommended statin and antiplatelet } \\
\text { therapy along with an educational bulletin } \\
\text { aimed to improve appropriate prescribing. } \\
\text { Prescribers }(\mathrm{n}=48) \text { received academic detailing } \\
\text { in addition to all mailed materials. }\end{array}$ & $\begin{array}{l}3 \% \text { increase in statin use and } 1 \% \text { increase in anticoag- } \\
\text { ulant use among CVD patients both groups (between } \\
\text { group } P=N S \text { ). } \\
5 \% \text { increase statin use in mailed only and } 4 \% \text { increase } \\
\text { in mailed + academic detailing among diabetic patients } \\
\text { (between group } P=N S \text { ). } \\
3 \% \text { increase in anticoagulant use in mailed only and } \\
2 \% \text { increase in mailed + academic detailing among } \\
\text { diabetic patients (between group } P=N S \text { ). }\end{array}$ \\
\hline O'Connell et al. (1999)28 & $\begin{array}{l}\text { ACE inhibitors, } \\
\text { antilipidemic } \\
\text { agents, H2RA, } \\
\text { NSAIDS, and oral } \\
\text { antibiotics }\end{array}$ & $\begin{array}{l}\text { Prescribers }(n=1,294) \text { received } 2 \text { interven- } \\
\text { tion packets } 6 \text { months apart that contained } \\
\text { prescriber feedback displaying individual and } \\
\text { peer prescribing rates. The first intervention } \\
\text { included an educational newsletter on general } \\
\text { prescribing issues and the second included } \\
\text { information on antibiotic prescribing. } \\
\text { Prescribers }(n=1,146) \text { served as a control } \\
\text { group. }\end{array}$ & $\begin{array}{l}\text { No significant differences were observed in prescrib- } \\
\text { ing patterns of any individual class of medications } \\
(P=N S) \text {. } \\
\text { For all target agents, prescribing rates per } 100 \\
\text { Medicare services per month increased from } 77.7 \text { to } \\
79.2 \text { in intervention and decreased from } 77.8 \text { to } 77.6 \\
\text { in control }(P=N S) \text {. }\end{array}$ \\
\hline $\begin{array}{l}\text { Okano and Rascati } \\
(1995)^{18}\end{array}$ & $\begin{array}{l}\text { Cimitidine, famoti- } \\
\text { dine, nizatidine, } \\
\text { ranitidine, omepra- } \\
\text { zole, sucralfate }\end{array}$ & $\begin{array}{l}\text { Physicians }(n=97) \text { received intervention pack- } \\
\text { ets regarding patients }(n=117) \text { who received } \\
\text { duplicate antiulcer therapy. Packets contained } \\
\text { a letter stating there is no evidence support- } \\
\text { ing concurrent use of an H2RA or omeprazole } \\
\text { with sucralfate and a profile for each identified } \\
\text { patient. } \\
\text { Patients ( } n=105 ; n=93 \text { physicians) served as a } \\
\text { control group. }\end{array}$ & $\begin{array}{l}55(57.9 \%) \text { of patients in the intervention group con- } \\
\text { tinued to receive concurrent therapy as compared with } \\
73(75.3 \%) \text { in the control group }(P=0.011) \text {. }\end{array}$ \\
\hline Owens et al. (2008)50 & $\begin{array}{l}\text { Oral triptans, ami- } \\
\text { triptyline, atenolol, } \\
\text { divalproex sodium, } \\
\text { metoprolol, pro- } \\
\text { pranolol, timolol, } \\
\text { topiramate, gaba- } \\
\text { pentin, verapamil }\end{array}$ & $\begin{array}{l}\text { Prescribers of patients }(\mathrm{n}=154) \text { identified as } \\
\text { potential candidates for prophylactic migraine } \\
\text { therapy received a cover letter describing the } \\
\text { program, an educational leaflet, and a response } \\
\text { form. }\end{array}$ & $\begin{array}{l}27(24.8 \%) \text { patients received a claim for a prophylactic } \\
\text { agent. } \\
91(83.5 \%) \text { patients had } \geq 1 \text { office visit. }\end{array}$ \\
\hline
\end{tabular}




\begin{tabular}{|c|c|c|c|}
\hline Study & Target Medications & $\begin{array}{l}\text { Program Description } \\
\text { and Intervention Materials }\end{array}$ & $\begin{array}{l}\text { Effects of Mailed Intervention } \\
\text { on Prescribing Behavior }\end{array}$ \\
\hline Pimlott et al. (2003) 29 & $\begin{array}{l}\text { Long- and short- } \\
\text { acting BDZ }\end{array}$ & $\begin{array}{l}\text { Physicians }(\mathrm{n}=168) \text { received prescriber feedback } \\
\text { displaying individual prescribing of } \mathrm{BDZ} \text { to } \\
\text { patients }>65 \text { years as compared with peers and } \\
\text { "best practice standards" as well as educational } \\
\text { information every } 2 \text { months for } 6 \text { months. } \\
\text { Physicians ( } \mathrm{n}=206 \text { ) received similar packets on } \\
\text { an unrelated topic and served as a control. }\end{array}$ & $\begin{array}{l}1.8(0.7 \%) \text { decrease in mean number of long-acting } \\
\text { BDZ in intervention increase of } 1.3(1.1 \%) \text { in control } \\
(P=0.036) \text {. } \\
1.6 \text { decrease in mean number of all BDZ prescriptions } \\
\text { in intervention and increase of } 5.8 \text { control }(P=\mathrm{NS}) \text {. } \\
\text { No difference in concurrent prescribing of BDZ and } \\
\text { other psychoactive agents }(P=N S) \text {. }\end{array}$ \\
\hline Raisch and Sleath (1999) 19 & $\begin{array}{l}\text { H2RAs, sucralfate, } \\
\text { other antiulcer } \\
\text { agents (not speci- } \\
\text { fied) }\end{array}$ & $\begin{array}{l}\text { Prescribers with } \geq 3 \text { patients }(\mathrm{n}=381) \text { identified } \\
\text { as recipients of potentially inappropriate anti- } \\
\text { ulcer therapy received a cover letter describing } \\
\text { the program, profiles of identified patients, and } \\
\text { educational material. } \\
\text { Half of the prescribers with } 1-2 \text { identified } \\
\text { received the intervention ( } \mathrm{n}=143 \text { prescribers } \\
\text { regarding } 156 \text { patients) and half served as a } \\
\text { control comparison group ( } \mathrm{n}=137 \text { prescribers } \\
\text { regarding } 156 \text { patients). }\end{array}$ & $\begin{array}{l}52(33.3 \%) \text { intervention and } 28(17.9 \%) \text { control } \\
\text { patients did not receive an antiulcer agent }(P<0.01) \text {. } \\
8(24.2 \%) \text { of intervention and } 6(23.1 \%) \text { control } \\
\text { patients had improvements in use of duplicate therapy } \\
(P=N S) \text {. } \\
11(9.8 \%) \text { intervention and } 0(0 \%) \text { control patients had } \\
\text { improvements in use without diagnosis }(P<0.01) \text {. } \\
52(47.3 \%) \text { intervention and } 24(24 \%) \text { control patients } \\
\text { had improvements in use of acute dose without diag- } \\
\text { nostic justification }(P<0.001) \text {. }\end{array}$ \\
\hline Rascati et al. (1996)20 & $\begin{array}{l}\text { cimitidine, famoti- } \\
\text { dine, nizatidine, } \\
\text { ranitidine, omepra- } \\
\text { zole, sucralfate }\end{array}$ & $\begin{array}{l}\text { Intervention packets that contained a letter } \\
\text { stating that literature does not support use } \\
\text { of duplicate antiulcer therapy and profiles of } \\
\text { identified patients }(n=174) \text { were sent to pre- } \\
\text { scribers }(n=184) \text {. } \\
\text { Patients }(n=161) \text { served as a control group. }\end{array}$ & $\begin{array}{l}61(47.7 \%) \text { intervention and } 85(64.4 \%) \text { control } \\
\text { patients continued to receive duplicate therapy } \\
(P=0.007) \text {. }\end{array}$ \\
\hline Rokstad et al. $(1995)^{30}$ & $\begin{array}{l}\text { BDZ, barbiturates, } \\
\text { antihistamines, } \\
\text { trimethoprim, } \\
\text { sulfonamides, } \\
\text { trimethoprim-sulfa, } \\
\text { extended spectrum } \\
\text { penicillins, nitrofu- } \\
\text { rantoin }\end{array}$ & $\begin{array}{l}\text { Physicians }(\mathrm{n}=51) \text { received prescriber feedback } \\
\text { displaying individual and peer prescribing } \\
\text { rates of target medications as well as expert } \\
\text { recommendations treatment of insomnia and } \\
\text { acute cystitis. } \\
\text { Physicians }(n=60) \text { served as a control group. }\end{array}$ & $\begin{array}{l}\text { Decrease in mean number of defined daily dose }{ }^{\mathrm{c}} \text { for } \\
\text { short-, medium-, and long-acting BDZ, BDZ tranquil- } \\
\text { izers, barbiturates, and increased use of antihistamines } \\
\text { and antidepressants in intervention group }(P<0.05 \text { for } \\
\text { all). No change in number of prescriptions for insom- } \\
\text { nia agents ( } P=\mathrm{NS} \text { ). } \\
\text { Decrease in mean number of defined daily dosec for } \\
\text { sulphonamides in intervention group }(P<0.05) \text {. No } \\
\text { change for other antibiotics ( } P=\mathrm{NS}) \text {. Increased number } \\
\text { of prescriptions for trimethroprim, decrease in number } \\
\text { of prescriptions for trimethoprim-sulfa in intervention } \\
\text { group }(P<0.05) \text {. }\end{array}$ \\
\hline Schectman et al. (1995) 45 & Cimetidine & $\begin{array}{l}\text { Physicians }(\mathrm{n}=63) \text { received an educational } \\
\text { memorandum followed by a "reminder memo" } \\
\text { alone or in addition to prescriber feedback dis- } \\
\text { playing individual H2RA prescribing patterns. } \\
\text { Physicians }(\mathrm{n}=12) \text { were excluded from the } \\
\text { intervention and used to check for secular } \\
\text { trends. }\end{array}$ & $\begin{array}{l}\text { Proportion of new cimetidine prescriptions increased } \\
\text { from } 21 \% \text { at baseline to } 30 \%(P<0.001) \text { in intervention } \\
\text { and decreased from } 21.6 \% \text { to } 17.6 \% \text { in excluded group } \\
(P=N S) \text {. } \\
\text { No difference between mailed education + feedback } \\
\text { and mailed education alone }(P=N S) \text {. }\end{array}$ \\
\hline Seltzer et al. (2000)21 & $\begin{array}{l}\text { BDZ, barbiturates, } \\
\text { acetylcarbromal, } \\
\text { chloral hydrate, } \\
\text { ethclorvynol, } \\
\text { ethinamate, glu- } \\
\text { tethimide, methyp- } \\
\text { rylon, paraldehyde, } \\
\text { zolpidem }\end{array}$ & $\begin{array}{l}\text { Physicians }(n=291) \text { with patients }(n=244) \\
\text { identified as receiving potentially excessive } \\
\text { sedative hypnotic therapy received a patient- } \\
\text { identifying letter that described guidelines for } \\
\text { sedative hypnotic use. }\end{array}$ & $\begin{array}{l}37(47.4 \%) \text { patients with responding prescribers who } \\
\text { indicated an intent to modify therapy no longer had } \\
\text { claims for sedative/hypnotics. }\end{array}$ \\
\hline Shatin et al. $(2005)^{51}$ & $\begin{array}{l}\text { Tramadol, selective } \\
\text { serotonin reuptake } \\
\text { inhibitors, tricyclic } \\
\text { antidepressants }\end{array}$ & $\begin{array}{l}\text { A single Dear Health Professional letter regard- } \\
\text { ing co-prescribing of tramadol and antidepres- } \\
\text { sants was sent to prescribers. }\end{array}$ & $\begin{array}{l}\text { Number of patients with a claim for an antidepressant } \\
\text { within } 30 \text { days of a first tramadol prescription claim } \\
\text { decreased from } 1,061(22.2 \%) \text { pre-intervention to } 844 \\
(19.0 \%) \text { post-intervention }(P=N S) \text {. }\end{array}$ \\
\hline
\end{tabular}




\begin{tabular}{|c|c|c|c|}
\hline Study & Target Medications & $\begin{array}{l}\text { Program Description } \\
\text { and Intervention Materials }\end{array}$ & $\begin{array}{l}\text { Effects of Mailed Intervention } \\
\text { on Prescribing Behavior }\end{array}$ \\
\hline Sleath et al. (1997)22 & $\begin{array}{l}\text { Sedatives (not } \\
\text { specified) }\end{array}$ & $\begin{array}{l}\text { Physicians }(n=211) \text { received educational infor- } \\
\text { mation and prescription and medical profiles } \\
\text { for patients }(n=269) \text { identified as recipients of } \\
\text { long-term sedative therapy. }\end{array}$ & $\begin{array}{l}49 \% \text { of patients with physicians who responded } \\
\text { to intervention with intent to change therapy had } \\
\text { a change in therapy. } 40 \% \text { of patients with physi- } \\
\text { cians who responded to intervention with no intent } \\
\text { to change therapy had a change in therapy. } 35 \% \text { of } \\
\text { patients with a physician who did not respond to } \\
\text { intervention had a change in therapy. }\end{array}$ \\
\hline Smith et al. (1998)23 & $\begin{array}{l}\text { Triazolam, temaze- } \\
\text { pam, estazolam, } \\
\text { quazepam, fluraz- } \\
\text { epam } \\
\text { Nontarget alterna- } \\
\text { tive medications: } \\
\text { other BDZ (i.e. } \\
\text { diazepam), bar- } \\
\text { biturates, chloral } \\
\text { hydrate }\end{array}$ & $\begin{array}{l}\text { Physicians and/or pharmacists involved in the } \\
\text { care of patients }(n=99) \text { identified as recipi- } \\
\text { ents of long-term sedative hypnotic therapy } \\
\text { received a cover letter, guidelines for sedative } \\
\text { hypnotic use, patient profiles, and prescriber } \\
\text { feedback displaying individual and peer pre- } \\
\text { scribing rates of target agents (pharmacists did } \\
\text { not receive prescriber feedback). } \\
\text { Patients ( } n=89 \text { ) served as a control group. }\end{array}$ & $\begin{array}{l}\text { Mean dosed decreased by } 8.1(27.6 \%) \text { in intervention } \\
\text { and } 2.4(8.5 \%) \text { control }(P=0.04) \text {. } \\
\text { Mean number of prescriptions decreased by } 0.9 \\
(26.5 \%) \text { in intervention and } 0.1(2.9 \%) \text { in control } \\
(P=0.004) \text {. } \\
\text { Mean number of tablets per prescription decreased by } \\
5.5(13.4 \%) \text { in intervention and } 2.9(7.2 \%) \text { in control } \\
(P=\mathrm{NS}) \text {. } \\
11 \text { intervention and } 4 \text { control patients discontinued } \\
\text { therapy with a target agent ( } P=\text { NS). } \\
\text { More patients in the intervention group began using } \\
\text { sedating antihistamines as compared with control }(6 \\
\text { [6.8\%] vs. } 1[1.3 \%] ; P=0.08) \text { and nontarget benzodiaz- } \\
\text { epines ( } 8[9.4 \%] \text { vs. } 0 \text { [0\%]; } P=0.01) \text {. }\end{array}$ \\
\hline Søndergaard et al. (2002)6 & $\begin{array}{l}\text { SABA, inhaled } \\
\text { corticosteroids }\end{array}$ & $\begin{array}{l}\text { Physicians received asthma management } \\
\text { guidelines and prescriber feedback displaying } \\
\text { number of patients using various amounts of } \\
\text { SABA and inhaled corticosteroids }(n=77) \text { or } \\
\text { number of patients who received a SABA as } \\
\text { compared to other area practices }(n=74) \text {. } \\
\text { Physicians }(n=141) \text { received information on } \\
\text { an unrelated topic and served as a control } \\
\text { group. } \\
\text { All groups received } 3 \text { interventions, sent } \\
3 \text { months apart. }\end{array}$ & $\begin{array}{l}\text { Neither patient count data }(\mathrm{HR}=0.77 ; P=\mathrm{NS}) \text { nor } \\
\text { aggregate feedback }(\mathrm{HR}=0.79 ; P=\mathrm{NS}) \text { had an impact } \\
\text { on prescribing inhaled corticosteroids to repeat SABA } \\
\text { users. } \\
\text { Neither patient count data }(\mathrm{HR}=0.1 .08 ; P=\mathrm{NS}) \text { nor } \\
\text { aggregate feedback (HR=0.92;P=NS) had an impact } \\
\text { on prescribing inhaled corticosteroids to first-time } \\
\text { SABA users. }\end{array}$ \\
\hline Søndergaard et al. (2003)5 & $\begin{array}{l}\text { Narrow spectrum } \\
\text { penicillin (penicil- } \\
\text { lin V), broad spec- } \\
\text { trum penicillins, } \\
\text { macrolides, fluoro- } \\
\text { quinolones }\end{array}$ & $\begin{array}{l}\text { Physicians }(\mathrm{n}=155) \text { received educational } \\
\text { material on diagnosis and treatment of upper } \\
\text { respiratory infections and prescriber feedback } \\
\text { displaying individual prescribing rates of anti- } \\
\text { biotics as compared with peers. } \\
\text { Physicians }(n=144) \text { received mailed education- } \\
\text { al material alone and served as a control group. }\end{array}$ & $\begin{array}{l}0.6 \% \text { decrease in prescribing rate of all antibiotics in } \\
\text { intervention as compared with control }(P=N S) \text {. } \\
0.02 \% \text { increase in fraction of narrow-spectrum peni- } \\
\text { cillin prescriptions in intervention as compared with } \\
\text { control }(P=N S) \text {. }\end{array}$ \\
\hline Starner et al. (2009)24 & $\begin{array}{l}\text { Determined accord- } \\
\text { ing to National } \\
\text { Committee for } \\
\text { Quality Assurance } \\
\text { list of drugs to } \\
\text { avoid in elderly }\end{array}$ & $\begin{array}{l}\text { 7,963 intervention letters alerting physicians of } \\
\text { PIP received by patients }(n=13,198) \geq 65 \text { years. }\end{array}$ & $\begin{array}{l}5,403(48.8 \%) \text { target medications claims were defined } \\
\text { as discontinued. }\end{array}$ \\
\hline Vaegter et al. (2010) ${ }^{43}$ & $\begin{array}{l}\text { Antacids, anti- } \\
\text { diabetic drugs, } \\
\text { cardiac disease } \\
\text { drugs, diuretics, } \\
\text { beta-blockers, CCB, } \\
\text { sex hormones, anti- } \\
\text { biotics, NSAIDs, } \\
\text { analgesics, neuro- } \\
\text { leptics, antidepres- } \\
\text { sants, asthma man- } \\
\text { agement agents }\end{array}$ & $\begin{array}{l}\text { Physicians }(\mathrm{n}=166) \text { received prescriber feed- } \\
\text { back displaying individual prescribing rates } \\
\text { and prescribing percentile as compared with } \\
\text { other practices for each of the } 13 \text { target classes. } \\
\text { Feedback was sent every } 6 \text { months for } 7 \text { years. }\end{array}$ & $\begin{array}{l}\text { Little variance was observed among prescribing rates } \\
\text { within practices, and large variations were noted } \\
\text { between practices. } \\
\text { Overall, intervention had no impact on prescribing } \\
\text { patterns. }\end{array}$ \\
\hline
\end{tabular}




\begin{tabular}{|c|c|c|c|}
\hline Study & Target Medications & $\begin{array}{l}\text { Program Description } \\
\text { and Intervention Materials }\end{array}$ & $\begin{array}{l}\text { Effects of Mailed Intervention } \\
\text { on Prescribing Behavior }\end{array}$ \\
\hline Woodward et al. $(2008)^{7}$ & $\begin{array}{l}\text { Polypharmacy: all } \\
\text { medications } \\
\text { Drug interaction: } \\
\text { all medications } \\
\text { excluding those } \\
\text { used on a short- } \\
\text { term basis }\end{array}$ & $\begin{array}{l}\text { Prescribers of patients }(n=81) \text { identified as } \\
\text { receiving polypharmacy or a potential drug } \\
\text { interaction }(n=836) \text { received a cover letter, } \\
\text { patient profile, and practice guidelines. }\end{array}$ & $\begin{array}{l}\text { Mean reduction of } 2.2 \text { unique medications and } 4.67 \\
\text { claims per polypharmacy patient }(P<0.05 \text { for both). } \\
\text { Mean reduction of } 0.73 \text { drug interaction pairs per } \\
\text { patient }(P<0.001) \text {. }\end{array}$ \\
\hline Zimmerman et al. (1994)11 & H2RA & $\begin{array}{l}\text { Prescribers with }>3 \text { patients ( } \mathrm{n}=195 \text { long- } \\
\text { term care; } \mathrm{n}=244 \text { ambulatory) identified } \\
\text { as recipients of high-dose long-term H2RA } \\
\text { therapy received a cover letter stating concerns, } \\
\text { journal references, a list of identified patients, } \\
\text { and H2RA history profile for each identified } \\
\text { patient. } \\
\text { Prescribers with } 3 \text { identified patients ( } \mathrm{n}=538 \\
\text { long-term care; } \mathrm{n}=1,202 \text { ambulatory) served as } \\
\text { a comparison group. }\end{array}$ & $\begin{array}{l}\text { No difference in mean [SD] monthly H2RA dose } \\
\text { months post-intervention as compared with com- } \\
\text { parison in long-term care }(15,575[12,518] \text { vs. } 16,722 \\
[9,957]) \text { or ambulatory }(17,133[10,801] \text { vs. } 18,685 \\
[11,340]) \text { patients ( } P=\text { NS for both). } \\
\text { Percent change greater in long-term care intervention } \\
\text { at } 6 \text { months ( } 40.2 \% \text { vs. } 31.8 \% ; P<0.05) \text { but not over } \\
12 \text {-month post-intervention period }(41.6 \% \text { vs. } 35.8 \% \text {; } \\
P=\text { NS). Percent change over } 12 \text {-month post-interven- } \\
\text { tion period greater in intervention than control }(36.8 \% \\
\text { vs. } 27.7 \% \text {; } P<0.01) \text {. } \\
\text { No change in hospitalization rates for ulcer recurrence } \\
\text { or gastrointestinal bleeding from baseline in either } \\
\text { population }(P=N S) \text {. }\end{array}$ \\
\hline Zuckerman et al. (2004)25 & Beta-blockers & $\begin{array}{l}\text { Prescribers }(\mathrm{n}=157 \text { ) received a cover letter, } \\
\text { an educational newsletter offering continuing } \\
\text { medical education credits, a list of post-AMI } \\
\text { patients who were potentially nonadherent } \\
\text { to beta-blocker therapy, and profiles for each } \\
\text { identified patient. } \\
\text { Prescribers ( } \mathrm{n}=328 \text { ) received mailed interven- } \\
\text { tion packets regarding post-AMI patients with- } \\
\text { out a claim for a beta-blocker. } \\
\text { Prescribers ( } \mathrm{n}=10,972) \text { expected to treat post- } \\
\text { AMI patients (excluding cardiologists) received } \\
\text { the educational newsletter. }\end{array}$ & $\begin{array}{l}\text { Beta-blocker use after } 7 \text { days increased from } 46.4 \% \text { to } \\
49.6 \% \text { and from } 61.3 \% \text { to } 64.7 \% \text { after } 30 \text { days }(P=N S) \text {. } \\
\text { Mailed intervention was associated with increased } \\
\text { likelihood of being prescribed a beta-blocker by } 16 \% \\
(P<0.01) \text {. } \\
\text { Percent of patients with beta-blocker adherence } \geq 80 \% \\
\text { increased from } 64.1 \% \text { to } 69.4 \%(P=0.02) \text {. }\end{array}$ \\
\hline Zuckerman et al. (2004)12 & Acetaminophen & $\begin{array}{l}\text { Physicians ( } \mathrm{n}=833 \text { ) received intervention con- } \\
\text { cerning patients }(\mathrm{n}=624) \text { identified as high- } \\
\text { dose acetaminophen users. Packets contained } \\
\text { a personalized cover letter, patient medication } \\
\text { profiles, a list of prescription and nonprescrip- } \\
\text { tion medications that contain acetaminophen, } \\
\text { and a medication reconciliation form for } \\
\text { patients. } \\
\text { Patients ( } \mathrm{n}=590) \text { identified as high-dose users } \\
\text { identified during a previous period composed a } \\
\text { historical control group. In addition acetamin- } \\
\text { ophen use was also compared with the entire } \\
\text { Medicaid population. }\end{array}$ & $\begin{array}{l}\text { Mean number of acetaminophen claims per patient } \\
\text { decreased from } 13.3 \text { to } 9.3 \text { for high-dose users. } \\
(P<0.001) \text {. This was an average of } 1 \text { claim decline } \\
\text { more than historical control group }(P=0.04) \text {. } \\
\text { Mean daily acetaminophen dose per patient decreased } \\
\text { from } 4.62 \text { grams to } 3.23 \text { grams for high-dose users } \\
(P<0.001) \text {. Similar decline noted in historical control } \\
\text { group }(P=N S) \text {. } \\
\text { Percent of patients with } \geq 2 \text { acetaminophen claims } \\
\text { decreased from } 65.0 \% \text { to } 32.5 \% \text { for high dose users } \\
(P<0.001) \text { Similar decline noted in historical control } \\
\text { group ( } P=\text { NS). } \\
\text { During the same period, there was a } 9 \% \text { decline in the } \\
\text { proportion of high-dose acetaminophen uses among } \\
\text { the entire Medicaid population and no difference in } \\
\text { the number of claims or average daily dose. }\end{array}$ \\
\hline
\end{tabular}

aAs measured by standardized normal therapeutic equivalent: ratio of total dose per 30 days to published compendia standards for usual daily dose for 30 days.

${ }^{b}$ As measured by real marginal value. Real marginal value was used to control for seasonal variations in use. Real marginal value = mean value in comparison population-each individual's mean value.

'Defined daily dose: assumed average daily dose for primary indication.

${ }^{d}$ As measured in triazolam milligram equivalents.

${ }^{e}$ As measured in cimetidine milligram equivalents.

$A C E=$ angiotensin-converting-enzyme; $A M I=$ acute myocardial infarction; $B D Z=$ benzodiazepines; $C C B=$ calcium channel blockers; $C I=$ confidence interval; $C V D=$ cardiovascular disease; H2RA = histamine H2 receptor antagonists; HMG-CoA=3-hydroxy-3-methyl-glutaryl-CoA; HR=hazard ratio; NS =nonsignificant; NSAIDS = non-steroidal anti-inflammatory drugs; $O R=$ odds ratio; $P C P=$ primary care provider; $P I P=$ potentially inappropriate prescribing; $S A B A=$ short-acting beta2-agonist; $S D=$ standard deviation. 
conferences. The potential benefit of academic outreach in addition to a mailed intervention is controversial; Avorn and Somerai $(1983)^{31}$ noted added improvement among recipients who received outreach visits, while Anderson et $a . .^{26}$ and Naughton et al. (2007) $)^{32}$ reported no added benefits.

A smaller subset of programs $(n=10)$ provided prescriber feedback in lieu of patient-identifying data., ${ }^{5,6,26-30,32,43,45}$ Typically, feedback displayed individual prescribing patterns relative to that of peer prescribers. Pimlott et al. (2003) also provided a comparison to "best practice standards." ${ }^{29}$ Schectman et al. (1995) was the only program that did not provide comparative data, as the authors believed the overall low volume of cimetidine use would discourage rather than encourage prescribing. ${ }^{45}$

The impact of providing prescriber feedback alone appears to be minimal. ${ }^{6,43}$ Søndergaard et al. (2002) found neither feedback presented as patient count data or in the form of a comparative aggregate graph had an impact on prescribing patterns. ${ }^{6}$ Even when combined with educational material or academic detailing, the impact is questionable: Of the 9 programs $^{5,23,26-30,32,45}$ that used prescriber feedback in combination with other techniques, 4 reported minimal to no improvements in prescribing patterns. ${ }^{5,28,29,32}$ Additionally, each of the 4 programs that provided educational materials alone were found to have little to no impact on measured outcomes. ${ }^{24,44,50,51}$ It should be noted that one program that reported positive outcomes used prescriber feedback in combination with patientidentifying data and educational material. ${ }^{23}$

Along with providing educational material for prescribers, several programs also included supplemental educational material for patients. ${ }^{3,10,12,22,29,31}$ With the exception of Jing et al. ${ }^{3}$ (who mailed material directly to patients), supplemental patient material was supplied as part of the prescriber's intervention packet. Patient-targeted materials ranged from tips regarding medication use and/or disease-state management to fill-in-the blank sheets for monitoring symptoms and medication reconciliation. Providing patient education material appears to contribute to intervention success, since the majority of programs that provided patient education materials were associated with significant improvements in prescribing behavior. ${ }^{3,10,12,22}$

Frequency of intervention mailings appears to be a secondary factor of importance relative to target selection and the provision of patient-identifying data. Continuous mailings of educational material or prescriber feedback alone often had minimal impact on prescribing patterns. ${ }^{6,31,44,45}$ Conversely, multiple mailings that contained patient-identifying data and/or a combination of intervention materials had a higher success rate. ${ }^{2-4,16,27,31}$ Among these, two programs found mailings sent on a quarterly basis to be associated with continuous significant improvements in prescribing throughout the entire study period. ${ }^{3,4}$

As displayed in Table 3, 20 of the identified programs included a response form requesting recipient feedback regarding the intervention. Response rate among reporting programs ranged from $25 \%$ to nearly $90 \%$. $2,9,10,12,14,18,20,21,27,29,32,46,48-50$ Generally, recipients viewed the intervention positively and often indicated intent to alter therapy based on the materials received. The majority of programs that reassessed patient profiles following response from mailings reported that an actual change in therapy occurred in approximately $50 \%$ of patients whose prescriber indicated intent to modify treatment. ${ }^{8,21,22,49}$ Exceptions to this finding include Culbertson et al., ${ }^{14}$ who reported an average of $14.3 \%$ change among their 3 study groups, and Okano and Rascati ${ }^{18}$ and Rascati et al. (1996), ${ }^{20}$ who reported changes of $73.3 \%$ and $81.2 \%$, respectively.

\section{Impact of Intervention}

Effect on Prescribing Patterns. With the exception of 2 programs, the authors concurred with individual program interpretation of impact on prescribing patterns (Table 2). Of the 15 programs that used a randomized controlled design to measure impact on prescribing patterns, 8 (53.3\%) reported a positive impact on at least 1 target outcome., , $^{2,20,23,26,27,31}$ Of the 21 remaining programs that used quasi-experimental or other observational designs, 18 (85.7\%) reported positive results. ${ }^{3,4,8-16,19,21,25,30,45,48,50}$

Exceptions to agreement with author interpretation include Owens et al. $(2008)^{50}$ and Starner et al. (2009). ${ }^{24}$ Though the intervention conducted by Owens et al. was associated with an increase use of prophylactic therapy, this study relied heavily on surrogate markers and assumptions regarding the indicated use of target agents and was classified as questionable impact for the purpose of this review..$^{50}$ Starner et al. found their intervention to be associated with a significant decrease in target agent use; however, this was determined according to prescriptions claims data obtained on a single day 6 months post-intervention. This method was relatively restrictive (as compared with that employed by other programs) and therefore may not be representative of actual prescribing patterns. ${ }^{24}$

Effect on Health Care Utilization. In addition to measuring effect on prescribing patterns, 5 programs also evaluated intervention impact on number of office visits, emergency room visits, and hospitalizations (Table 2).8,10,11,22,50 None of the programs noted changes in emergency room visits or hospitalizations. Increased office visits were observed by Sleath et al. (1997) and Owens et al., while Coleman et al. (2003) noted decreased office visits and use of physician services among both intervention and comparison groups. ${ }^{8,22,50}$

Economic Impact. Table 4 displays the association between mailed intervention and change in economic outcomes. While most programs reported a downward trend in prescription costs, they often failed to reach statistical significance. Sleath et al. was unique in that they noted that an upward trend in costs 


\section{TABLE 3 Assessment of Prescriber Response}

\begin{tabular}{|c|c|c|c|c|}
\hline Study & Form Objectives & $\begin{array}{c}\text { Response } \\
\text { Rate }\end{array}$ & Prescriber Response & $\begin{array}{l}\text { Association Between } \\
\text { Response and Change in } \\
\text { Prescribing Pattern }\end{array}$ \\
\hline Bjornson et al. (1990)49 & $\begin{array}{l}\text { Assess awareness of pub- } \\
\text { lished study. } \\
\text { Assess intent to alter therapy } \\
\text { based on study results. } \\
\text { Identify additional factors } \\
\text { that may influence decision } \\
\text { to modify therapy. }\end{array}$ & $\begin{array}{l}53.1 \% \\
(152 / 286) ; \\
49.3 \% \\
\text { (141/286) } \\
\text { usable }\end{array}$ & $\begin{array}{l}95(67.4 \%) \text { of respondents indicate they } \\
\text { were aware of the study clinical trial. } \\
35.5 \% \text { (50) intended to alter prescribing } \\
\text { based on clinical trial results; } 24.1 \% \text { (38) } \\
\text { did not intend to alter therapy; } 19.1 \% \\
\text { (28) indicated decision would be patient } \\
\text { dependent. } \\
\text { Key factors include drug availability, } \\
\text { professional training, experience with } \\
\text { target drug's adverse event, size of } \\
\text { mortality reduction, and comments by } \\
\text { peers. }\end{array}$ & $\begin{array}{l}\text { Slightly over one-half of patients } \\
\text { with a responding physician who } \\
\text { indicated intent to change had a } \\
\text { full or partial change in therapy. }\end{array}$ \\
\hline Coleman et al. (2003) 46 & $\begin{array}{l}\text { Assess intent to intervene. } \\
\text { Assess potential incorrect } \\
\text { identification of prescribers } \\
\text { or pharmacists. }\end{array}$ & $\begin{array}{l}47.3 \% \\
(43 / 91)\end{array}$ & $\begin{array}{l}21(48.8 \%) \text { intended to intervene. } \\
6(13.9 \%) \text { indicated previous unsuccess- } \\
\text { ful attempt. } \\
6(13.9 \%) \text { did not intend to not inter- } \\
\text { vene. } \\
10(23.3 \%) \text { reported incorrect provider. }\end{array}$ & $\begin{array}{l}47.6 \%(10 / 21) \text { of patients with } \\
\text { physicians who indicated intent to } \\
\text { intervene had a change in therapy. }\end{array}$ \\
\hline Culbertson et al. (1999)14 & $\begin{array}{l}\text { Assess intent to modify } \\
\text { therapy. }\end{array}$ & $\begin{array}{l}44.8 \% \\
(205 / 458)\end{array}$ & $\begin{array}{l}\text { Prescriber only: } 23 \% \text { reported intent to } \\
\text { change therapy. } \\
\text { Prescriber + pharmacist mailed inter- } \\
\text { vention: } 35 \% \text { reported intent to change } \\
\text { therapy. } \\
\text { Prescriber + pharmacist mailed inter- } \\
\text { vention + phone call: } 44 \% \text { reported } \\
\text { intent to change therapy. }\end{array}$ & $\begin{array}{l}\text { Prescriber only: } 11 \% \text { made change } \\
\text { in therapy. } \\
\text { Prescriber + pharmacist mailed } \\
\text { intervention: } 13 \% \text { made change in } \\
\text { therapy. } \\
\text { Prescriber + pharmacist mailed } \\
\text { intervention + phone call: } 19 \% \\
\text { made changed in therapy. }\end{array}$ \\
\hline Fick et al. $(2004)^{2}$ & $\begin{array}{l}\text { Assess intent to modify } \\
\text { therapy. } \\
\text { Assess potential incorrect } \\
\text { identification of prescribers. }\end{array}$ & $\begin{array}{l}71.2 \% \\
(84 / 118)\end{array}$ & $\begin{array}{l}211(12.5 \%) \text { indicated discontinuation of } \\
\text { medication. } \\
28(1.7 \%) \text { indicated decreased dose or } \\
\text { frequency. } \\
21(1.2 \%) \text { indicated prescribed an alter- } \\
\text { native medication. } \\
1,327(78.4 \%) \text { indicated assessed patient } \\
\text { with no change. } \\
105(6.2 \%) \text { indicated other response. }\end{array}$ & NR \\
\hline Guo et al. (1995) ${ }^{9}$ & $\begin{array}{l}\text { Assess general comments } \\
\text { regarding intervention. }\end{array}$ & $\begin{array}{l}68.6 \% \\
(81 / 118)\end{array}$ & NR & NR \\
\hline Hux et al. (1999)27 & $\begin{array}{l}\text { Assess perceived value of } \\
\text { intervention. } \\
\text { Assess acceptability of } \\
\text { intervention. }\end{array}$ & $\begin{array}{l}76.0 \% \\
(76 / 100)\end{array}$ & $\begin{array}{l}\text { Respondents rated each of the following } \\
\text { measures of data effectiveness as } 4 / 5 \text { : } \\
\text { usefulness of feedback as an education } \\
\text { tool, impact on prescribing, motivation } \\
\text { to learn about appropriate antibiotic use, } \\
\text { willingness to participate in a similar } \\
\text { program again. } \\
\text { Factors most contributing to the accept- } \\
\text { ability of an intervention included } \\
\text { confidentially and data prepared apart } \\
\text { from payer. Secondary factors included } \\
\text { emphasis on efficacy rather than costs, } \\
\text { obtaining prescriber consent, and access } \\
\text { to program via fax. Tertiary factors } \\
\text { included provision of continuing medi- } \\
\text { cal education credits. }\end{array}$ & NR \\
\hline
\end{tabular}




\section{TABLE 3 Assessment of Prescriber Response (continued)}

\begin{tabular}{|c|c|c|c|c|}
\hline Study & Form Objectives & $\begin{array}{c}\text { Response } \\
\text { Rate }\end{array}$ & Prescriber Response & $\begin{array}{l}\text { Association Between } \\
\text { Response and Change in } \\
\text { Prescribing Pattern }\end{array}$ \\
\hline Lee et al. $(2004)^{10}$ & $\begin{array}{l}\text { Assess perceived value of } \\
\text { intervention. } \\
\text { Assess intent to modify } \\
\text { therapy. }\end{array}$ & $\begin{array}{l}44.1 \% \\
(249 / 564)\end{array}$ & $\begin{array}{l}197(50 \%) \text { felt the intervention provided } \\
\text { new information. } \\
189(70 \%) \text { intended to use the patient } \\
\text { education material provided. } \\
190(78 \%) \text { felt supplemental education } \\
\text { material will improve communication } \\
\text { with patients. } \\
185 \text { ( } 56 \%) \text { intended to modify therapy } \\
\text { for at least } 1 \text { patient. }\end{array}$ & NR \\
\hline Naughton et al. $(2007)^{32}$ & $\begin{array}{l}\text { Assess level of satisfaction } \\
\text { with intervention. } \\
\text { Assess willingness to contin- } \\
\text { ue receiving intervention. } \\
\text { Assess perceived impact on } \\
\text { practice. } \\
\text { Assess general comments } \\
\text { regarding intervention. }\end{array}$ & $\begin{array}{l}80.0 \% \\
(88 / 110) \\
\text { for mailed } \\
\text { intervention } \\
89.9 \% \\
(48 / 54) \\
\text { for academic } \\
\text { detailing }\end{array}$ & $\begin{array}{l}\text { Both groups expressed a high level of } \\
\text { satisfaction with intervention, and there } \\
\text { were no between-group differences in } \\
\text { question responses ( } P=\mathrm{NS} \text { ). } \\
\text { All respondents who received postal } \\
\text { bulletin indicated they would like to } \\
\text { continue receiving mailed intervention, } \\
\text { and } 79(84 \%) \text { felt the feedback had } \\
\text { impact on their practice. } \\
39(81 \%) \text { receiving academic detailing } \\
\text { indicated they would like to continu- } \\
\text { ing receiving academic detailing with } \\
\text { mailed intervention. }\end{array}$ & NR \\
\hline $\begin{array}{l}\text { Okano and Rascati } \\
(1995)^{18}\end{array}$ & $\begin{array}{l}\text { Assess agreement/disagree- } \\
\text { ment with intervention. } \\
\text { Assess potential incorrect } \\
\text { identification of prescribers. } \\
\text { Assess general comments } \\
\text { regarding intervention. }\end{array}$ & $\begin{array}{l}67.5 \% \\
(79 / 117)\end{array}$ & $\begin{array}{l}39(49.4 \%) \text { agreed with the intervention. } \\
23(29.1 \%) \text { disagreed with the interven- } \\
\text { tion. } \\
8(10.1 \%) \text { reported not my patient. } \\
7(8.9 \%) \text { reported no longer seeing } \\
\text { patient. } \\
2(2.5 \%) \text { reported they did not prescribe } \\
\text { the medication. }\end{array}$ & $\begin{array}{l}\text { Profiles for } 30 \text { of the } 39 \text { patients } \\
\text { with physicians who agreed with } \\
\text { the intervention were available for } \\
\text { evaluation after } 6 \text { months. } \\
\text { Of these patients, } 73.3 \%(22 / 30) \\
\text { had a change in therapy. }\end{array}$ \\
\hline Owens et al. (2008)50 & $\begin{array}{l}\text { Assess perceived utility of } \\
\text { migraine prophylaxis. } \\
\text { Assess perceived value of } \\
\text { intervention. }\end{array}$ & $\begin{array}{l}25.2 \% \\
(41 / 163)\end{array}$ & $\begin{array}{l}\text { Approximately } 90 \% \text { believed prophy- } \\
\text { lactic therapy to be "very effective" or } \\
\text { "somewhat effective." } \\
\text { Approximately } 80 \% \text { indicated the inter- } \\
\text { vention was helpful. }\end{array}$ & NR \\
\hline Pimlott et al. $(2003)^{29}$ & $\begin{array}{l}\text { Assess level of satisfaction } \\
\text { with intervention. }\end{array}$ & $\begin{array}{l}48.2 \% \\
(81 / 168)\end{array}$ & $\begin{array}{l}70 \% \text { indicated they would participate in } \\
\text { a similar mailed intervention program. }\end{array}$ & NR \\
\hline Raisch and Sleath (1999)19 & $\begin{array}{l}\text { Assess intent to intervene } \\
\text { and intended method of } \\
\text { intervention. }\end{array}$ & NR & NR & NR \\
\hline Rascati et al. (1996)20 & $\begin{array}{l}\text { Assess agreement/disagree- } \\
\text { ment with intervention. } \\
\text { Assess potential incorrect } \\
\text { identification of prescribers. } \\
\text { Assess general comments } \\
\text { regarding intervention. }\end{array}$ & $\begin{array}{l}71.2 \% \\
(131 / 184)\end{array}$ & $\begin{array}{l}64(48.9 \%) \text { agreed with the interven- } \\
\text { tion. } \\
25(19.1 \%) \text { disagreed with the interven- } \\
\text { tion. } \\
13(9.9 \%) \text { reported not my patient. } \\
16(12.2 \%) \text { reported no longer seeing } \\
\text { patient. } \\
11(8.4 \%) \text { reported they did not pre- } \\
\text { scribe the medication. } \\
2(1.5 \%) \text { indicated "other" response. }\end{array}$ & $\begin{array}{l}\text { Profiles for } 44 \text { of the } 62 \text { patients } \\
\text { with physicians who agreed with } \\
\text { the intervention and } 21 \text { of the } 25 \\
\text { patient with physicians who dis- } \\
\text { agreed with the intervention were } \\
\text { available for evaluation. } \\
18.2 \% \text { (8/44) of patients with phy- } \\
\text { sicians who agreed continued to } \\
\text { receive duplicate therapy as com- } \\
\text { pared with } 80.9 \%(17 / 21) \text { among } \\
\text { patients with physicians who dis- } \\
\text { agreed }(P<0.001) \text {. }\end{array}$ \\
\hline
\end{tabular}




\section{TABLE 3 Assessment of Prescriber Response (continued)}

\begin{tabular}{|c|c|c|c|c|}
\hline Study & Form Objectives & $\begin{array}{l}\text { Response } \\
\text { Rate }\end{array}$ & Prescriber Response & $\begin{array}{l}\text { Association Between } \\
\text { Response and Change in } \\
\text { Prescribing Pattern }\end{array}$ \\
\hline Seltzer et al. $(2000)^{21}$ & $\begin{array}{l}\text { Assess agreement/disagree- } \\
\text { ment with intervention. } \\
\text { Assess potential incorrect } \\
\text { identification of prescribers. } \\
\text { Assess general comments } \\
\text { regarding intervention. }\end{array}$ & $\begin{array}{l}71.5 \% \\
(208 / 291)\end{array}$ & $\begin{array}{l}64(40.4 \%) \text { agreed with the interven- } \\
\text { tion. } \\
55(26.4 \%) \text { disagreed with the interven- } \\
\text { tion. } \\
18(8.7 \%) \text { reported not my patient. } \\
16(7.7 \%) \text { reported no longer seeing } \\
\text { patient. } \\
17(8.2 \%) \text { reported they did not pre- } \\
\text { scribe the medication. } \\
18(8.7 \%) \text { indicated "other" response. } \\
\text { Many physicians who disagreed or } \\
\text { selected "other" indicated that chronic } \\
\text { illness justified long-term treatment. }\end{array}$ & $\begin{array}{l}\text { Profiles for } 78 \text { of the } 84 \text { patients } \\
\text { with physicians who agreed with } \\
\text { the intervention were available for } \\
\text { evaluation. } \\
\text { Of these, } 47.4 \% \text { ( } 37 / 78 \text { ) discontin- } \\
\text { ued sedative/hypnotic therapy. }\end{array}$ \\
\hline Sleath et al. $(1997)^{22}$ & $\begin{array}{l}\text { Assess intent to intervene } \\
\text { and intended method of } \\
\text { intervention (i.e., counseling, } \\
\text { providing spacer, etc.). }\end{array}$ & NR & NR & NR \\
\hline Sleath and Collins (1997) ${ }^{48}$ & $\begin{array}{l}\text { Assess intent to intervene } \\
\text { and intended method of } \\
\text { intervention. } \\
\text { Assess potential incorrect } \\
\text { identification of prescribers. } \\
\text { Gage recipient tone regarding } \\
\text { intervention. }\end{array}$ & $\begin{array}{l}49 \% \text { of } \\
\text { physicians } \\
\text { returned } \\
\text { form regard- } \\
\text { ing } 47 \% \\
\text { (126) of } \\
\text { patients }\end{array}$ & $\begin{array}{l}24(19 \%) \text { reported an intent to change } \\
\text { therapy. } \\
48(38 \%) \text { intended to monitor or coun- } \\
\text { sel. } \\
21(17 \%) \text { intended to recommend non- } \\
\text { pharmacologic treatment. } \\
33(26 \%) \text { reported no planned action } \\
\text { due to patient demand. } \\
14 \text { ( } 11 \%) \text { reported no planned action } \\
\text { because they had incorrectly received } \\
\text { intervention. } \\
2 \% \text { of responses were positive in tone; } \\
92 \% \text { were neutral; and } 6 \% \text { were negative. }\end{array}$ & $\begin{array}{l}49 \% \text { of patients with physicians } \\
\text { who intended to act on interven- } \\
\text { tion had a change in therapy. } \\
40 \% \text { of patients with physicians } \\
\text { who planned no action had a } \\
\text { change in therapy. } \\
35 \% \text { of patients with physicians } \\
\text { who did not respond had a change } \\
\text { in therapy. }\end{array}$ \\
\hline Starner et al. (2009)24 & Assess acceptance of program. & NR & NR & NR \\
\hline Woodward et al. $(2008)^{7}$ & Assess acceptance of program. & NR & NR & NR \\
\hline Zuckerman et al. (2004)12 & Assess acceptance of program. & NR & NR & NR \\
\hline Zuckerman et al. (2004)25 & $\begin{array}{l}\text { Assess willingness to modify } \\
\text { therapy. }\end{array}$ & $\begin{array}{l}34.5 \% \\
(287 / 833)\end{array}$ & $\begin{array}{l}50 \% \text { were willing to change therapy for } \\
\text { at least } 1 \text { patient. } \\
30 \% \text { were unwilling to change therapy. } \\
20 \% \text { did not respond to question. }\end{array}$ & NR \\
\hline
\end{tabular}

NR=not reported .

of asthma-related health care utilization occurred in 1 of their 2 intervention groups. ${ }^{22}$ Though greater reductions were often observed among groups that received more intensive interventions, the added costs may result in a negligible net benefit.

Avorn et al. estimated the costs of their intervention, which incorporated both mailed materials and academic detailing, to be approximately $\$ 100$ per physician. ${ }^{31}$ With this taken into account, the net savings in target medications costs is reduced from $\$ 105$ to $\$ 5$ per physician. Similarly, Culbertson et al. noted additional costs decreased net prescription savings overall of their intensive intervention from $\$ 107$ to $\$ 52$ per patient. ${ }^{14}$

\section{Discussion}

Although evidence-based medicine is the cornerstone of today's clinical practice, successful treatment often relies on coordination of care between providers and patient adherence to prescribed therapies. Mailed interventions may promote coordination of care between prescribers and provide real-world information regarding patient prescription use. This review identified 40 publications that described the results of 39 distinct mailed intervention initiatives and links the effectiveness of letter interventions to various outcomes. Approximately 50\% of the randomized controlled trials reported a positive impact 


\section{TABLE 4 Economic Impact of Mailed Intervention}

\begin{tabular}{|c|c|c|c|c|c|c|c|c|c|c|c|c|c|c|}
\hline Study & $\begin{array}{l}\text { Mailed } \\
\text { Intervention } \\
\text { Materials }\end{array}$ & Effect Measure & \multicolumn{12}{|c|}{ Economic Impact of Mailed Intervention } \\
\hline \multirow{2}{*}{$\begin{array}{l}\text { Avorn } \\
\text { and } \\
\text { Soumerai } \\
(1983)^{31}\end{array}$} & \multirow{2}{*}{$\begin{array}{l}\text { Educational } \\
\text { material } \pm \text { sup- } \\
\text { plemental patient } \\
\text { education mate- } \\
\text { rial } \pm \text { academic } \\
\text { detailing }\end{array}$} & \multirow[t]{2}{*}{$\begin{array}{l}\text { Change in prescribing } \\
\text { costs per physician }(\$)\end{array}$} & \multirow{2}{*}{\multicolumn{4}{|c|}{$\begin{array}{l}\text { Academic Detailing + } \\
\text { Mailed Intervention }\end{array}$}} & \multicolumn{2}{|c|}{$\begin{array}{c}\text { Mailed } \\
\text { Intervention }\end{array}$} & \multicolumn{3}{|c|}{ Control } & \multicolumn{3}{|c|}{$P$-Value } \\
\hline & & & & & & & $\mathrm{N}$ & JR & & NR & & & 0.002 & \\
\hline \multirow{4}{*}{$\begin{array}{l}\text { Collins } \\
\text { et al. } \\
(1997)^{13}\end{array}$} & \multirow{4}{*}{$\begin{array}{l}\text { Patient profile } \\
\text { + educational } \\
\text { information }\end{array}$} & \multirow{3}{*}{$\begin{array}{l}\text { Change in mean [SD] } \\
\text { dipyridamole expen- } \\
\text { diture per long-term } \\
\text { care patient }(\$)\end{array}$} & \multicolumn{3}{|c|}{ Physician Only } & \multicolumn{3}{|c|}{ Pharmacist Only } & \multicolumn{3}{|c|}{ Physician + Pharmacist } & \multicolumn{3}{|c|}{ Control } \\
\hline & & & Pre & Post & $P$-Value & Pre & Post & $P$-Value & Pre & Post & $P$-Value & Pre & Post & $P$-Value \\
\hline & & & $\begin{array}{l}189.12 \\
{[72.52]}\end{array}$ & $\begin{array}{l}119.23 \\
{[107.19]}\end{array}$ & 0.007 & $\begin{array}{l}188.27 \\
{[77.29]}\end{array}$ & $\begin{array}{c}98.30 \\
{[98.01]}\end{array}$ & 0.007 & $\begin{array}{l}159.49 \\
{[77.50]}\end{array}$ & $\left.\begin{array}{c}62.92 \\
{[94.67]}\end{array}\right]$ & 0.007 & $\begin{array}{l}198.88 \\
{[68.91]}\end{array}$ & $\begin{array}{l}139.39 \\
{[110.73]}\end{array}$ & NS \\
\hline & & $\begin{array}{l}\text { Change in mean [SD] } \\
\text { dipyridamole expen- } \\
\text { diture per ambulatory } \\
\text { patient (\$) }\end{array}$ & $\begin{array}{l}122.62 \\
{[78.15]}\end{array}$ & $\begin{array}{c}84.69 \\
{[92.16]}\end{array}$ & NS & $\begin{array}{l}143.59 \\
{[78.49]}\end{array}$ & $\left.\mid \begin{array}{c}94.63 \\
{[90.92]}\end{array}\right]$ & NS & $\begin{array}{l}146.26 \\
{[85.63]}\end{array}$ & $\left.\begin{array}{c}85.05 \\
{[101.10]}\end{array}\right]$ & NS & $\begin{array}{l}142.72 \\
{[83.22]}\end{array}$ & $\begin{array}{r}114.78 \\
{[94.52]}\end{array}$ & NS \\
\hline \multirow{3}{*}{$\begin{array}{l}\text { Culbertson } \\
\text { et al. } \\
(1999)^{14}\end{array}$} & \multirow{3}{*}{$\begin{array}{l}\text { Patient profile } \\
\pm \text { educational } \\
\text { material }\end{array}$} & \multirow{2}{*}{$\begin{array}{l}\text { Mean change in cost } \\
\text { of antiulcer medica- } \\
\text { tions per patient }(\$)\end{array}$} & \multicolumn{4}{|c|}{ Physician Only } & \multicolumn{4}{|c|}{ Physician + Pharmacist } & \multicolumn{4}{|c|}{$\begin{array}{c}\text { Physician + Pharmacist + } \\
\text { Follow-Up Call }\end{array}$} \\
\hline & & & \multicolumn{4}{|c|}{-55.87} & \multicolumn{4}{|c|}{-42.66} & \multicolumn{4}{|c|}{-107.75} \\
\hline & & $\begin{array}{l}\text { Impact of various } \\
\text { intervention tech- } \\
\text { niques on antiulcer } \\
\text { medication cost }\end{array}$ & \multicolumn{12}{|c|}{$\begin{array}{l}\text { Physician + pharmacist }+ \text { follow-up call group had greater decrease in cost before and after } \\
\text { intervention as compared with other study groups }(P<0.05) \text {. }\end{array}$} \\
\hline \multirow[t]{2}{*}{$\begin{array}{l}\text { Guo } \\
\text { et al. } \\
(1995)^{9}\end{array}$} & \multirow[t]{2}{*}{ Patient profile } & $\begin{array}{l}\text { Change drug } \\
\text { reimbursement as } \\
\text { measured by real } \\
\text { marginal value } \\
(\$)\end{array}$ & \multicolumn{12}{|c|}{$\begin{array}{l}\text { Significant downward trend in drug reimbursement throughout follow-up period }(P=0.002) \text {. } \\
\text { Decrease in drug reimbursement significant in } 5 \text { of the } 7 \text { follow-up months }(P<0.05) \text {. }\end{array}$} \\
\hline & & $\begin{array}{l}\text { Change in total drug } \\
\text { reimbursement per pre- } \\
\text { scriber per month }(\$)\end{array}$ & \multicolumn{12}{|c|}{-112.73} \\
\hline \multirow{3}{*}{$\begin{array}{l}\text { Hoffman } \\
\text { et al. } \\
(2003)^{16}\end{array}$} & \multirow[t]{3}{*}{ Patient profile } & $\begin{array}{l}\text { Change in mean [SD] } \\
\text { prescription drug }\end{array}$ & & Interv & ention & & & Con & ntrol & & & $P-V_{c}$ & Talue & \\
\hline & & $\begin{array}{l}\text { costs per member per } \\
\text { month }(\$)\end{array}$ & & -118.38 & [296.00] & & & -39.07[ & [331.00] & & & & NS & \\
\hline & & $\begin{array}{l}\text { Change in medical } \\
\text { costs per member per } \\
\text { year }(\$)\end{array}$ & & -1 & 413 & & & -9 & 970 & & & $<0$ & 0.05 & \\
\hline $\begin{array}{l}\text { Hux et al. } \\
(1999)^{27}\end{array}$ & $\begin{array}{l}\text { Prescriber } \\
\text { feedback }\end{array}$ & $\begin{array}{l}\text { Change in median } \\
\text { antibiotic cost }\end{array}$ & $\begin{array}{l}\text { Increas } \\
\text { change }\end{array}$ & $\begin{array}{l}\text { sed by a } \\
\text { e in inter }\end{array}$ & $\begin{array}{l}n \text { average } \\
\text { rvention }\end{array}$ & $\begin{array}{l}\text { e of over } \\
\text { group ( }\end{array}$ & $\begin{array}{l}\$ 3 \text { per } \\
P=0.002\end{array}$ & $\begin{array}{l}\text { prescrip } \\
\text { 2). }\end{array}$ & tion in c & control g & roup as & compare & ed with n & \\
\hline Sleath & Patient profile & & & & Physicia & an Only & & & & Phy & sician + & Pharma & acist & \\
\hline & + educational & & $\mathbf{P}$ & re & Po & ost & $P-\mathrm{Va}$ & alue & $\mathrm{Pr}$ & re & Po & ost & $P-V_{c}$ & Talue \\
\hline & & $\begin{array}{l}\text { Change in mean [SD] } \\
\text { cost of SABA (\$) }\end{array}$ & $\begin{array}{r}92 \\
{[47}\end{array}$ & .31 & $\begin{array}{r}82 . \\
{[84 .}\end{array}$ & $\begin{array}{l}.51 \\
.26]\end{array}$ & $\mathrm{N}$ & NS & $\begin{array}{r}74 \\
{[38}\end{array}$ & .96 & $\begin{array}{r}61 \\
{[37}\end{array}$ & $\begin{array}{l}.14 \\
7.12]\end{array}$ & & 0.05 \\
\hline & & $\begin{array}{l}\text { Change in mean [SD] } \\
\text { cost of asthma medi- } \\
\text { cations (\$) }\end{array}$ & $\begin{array}{l}157 \\
{[94}\end{array}$ & $\begin{array}{l}7.53 \\
.93]\end{array}$ & $\begin{array}{r}138 \\
{[124}\end{array}$ & $\begin{array}{l}8.55 \\
4.19]\end{array}$ & $\mathrm{N}$ & JS & $\begin{array}{l}152 \\
{[95}\end{array}$ & $\begin{array}{l}2.84 \\
83]\end{array}$ & $\begin{array}{l}132 \\
{[91 .}\end{array}$ & $\begin{array}{l}2.08 \\
.06]\end{array}$ & & 0.05 \\
\hline & & $\begin{array}{l}\text { Change in mean }[S D] \\
\text { cost of asthma-related } \\
\text { health care use (\$) }\end{array}$ & $\begin{array}{r}81 \\
{[344}\end{array}$ & $\begin{array}{l}.25 \\
4.44]\end{array}$ & $\begin{array}{r}229 \\
{[818}\end{array}$ & $\begin{array}{l}9.94 \\
8.12]\end{array}$ & $\mathrm{N}$ & JS & $\begin{array}{r}47 \\
{[93}\end{array}$ & $\begin{array}{l}.74 \\
.00]\end{array}$ & & .13 & & NS \\
\hline & & $\begin{array}{l}\text { Change in mean [SD] } \\
\text { cost of asthma medi- } \\
\text { cations and health } \\
\text { care use (\$) }\end{array}$ & $\begin{array}{r}238 \\
{[353}\end{array}$ & $\begin{array}{l}3.78 \\
3.88]\end{array}$ & $\begin{array}{r}361 \\
{[815}\end{array}$ & $\begin{array}{l}1.50 \\
5.21]\end{array}$ & $\mathrm{N}$ & NS & $\begin{array}{l}200 \\
{[137}\end{array}$ & $\begin{array}{l}0.58 \\
7.76]\end{array}$ & $\begin{array}{l}171 \\
{[127}\end{array}$ & $\begin{array}{l}1.21 \\
7.31]\end{array}$ & & NS \\
\hline
\end{tabular}


Improving the Success of Mailed Letter Intervention Programs to Influence Prescribing Behaviors: A Review

TABLE 4 Economic Impact of Mailed Intervention (continued)

\begin{tabular}{|c|c|c|c|c|c|c|c|c|c|c|c|}
\hline \multirow{5}{*}{$\begin{array}{l}\text { Study } \\
\text { Zimmerman } \\
\text { et al. } \\
(1994)^{11}\end{array}$} & \multirow{5}{*}{\begin{tabular}{l}
\multicolumn{1}{c}{$\begin{array}{c}\text { Mailed } \\
\text { Intervention } \\
\text { Materials }\end{array}$} \\
Patient profile \\
+ educational \\
material
\end{tabular}} & Effect Measure & \multicolumn{9}{|c|}{ Economic Impact of Mailed Intervention } \\
\hline & & & \multicolumn{3}{|c|}{ Intervention } & \multicolumn{3}{|c|}{ Comparison } & \multicolumn{3}{|c|}{$P$-Value } \\
\hline & & & Pre & $\begin{array}{c}6 \\
\text { Months } \\
\text { Post } \\
\end{array}$ & $\begin{array}{c}12 \\
\text { Months } \\
\text { Post } \\
\end{array}$ & Pre & $\begin{array}{c}6 \\
\text { Months } \\
\text { Post } \\
\end{array}$ & $\begin{array}{c}12 \\
\text { Months } \\
\text { Post } \\
\end{array}$ & Pre & $\begin{array}{c}6 \\
\text { Months } \\
\text { Post } \\
\end{array}$ & $\begin{array}{c}12 \\
\text { Months } \\
\text { Post }\end{array}$ \\
\hline & & $\begin{array}{l}\text { Change in mean }[S D] \\
\text { cost of H2RA in long- } \\
\text { term care population } \\
(\$)\end{array}$ & $\begin{array}{c}67.99 \\
{[12.04]}\end{array}$ & $\begin{array}{c}44.11 \\
{[32.25]}\end{array}$ & $\begin{array}{c}43.17 \\
{[31.97]}\end{array}$ & $\begin{array}{l}64.78 \\
{[14.99]}\end{array}$ & $\begin{array}{l}48.55 \\
{[25.50]}\end{array}$ & $\begin{array}{l}46.36 \\
{[27.62]}\end{array}$ & $<0.005$ & NS & NS \\
\hline & & $\begin{array}{l}\text { Change in mean [SD] } \\
\text { cost of H2RA } \\
\text { in ambulatory } \\
\text { population }(\$)\end{array}$ & $\begin{array}{c}60.29 \\
{[15.06]}\end{array}$ & $\begin{array}{c}44.69 \\
{[27.60]}\end{array}$ & $\begin{array}{c}43.64 \\
{[31.66]}\end{array}$ & $\begin{array}{l}57.63 \\
{[18.14]}\end{array}$ & $\begin{array}{c}47.86 \\
{[31.86]}\end{array}$ & $\begin{array}{l}46.05 \\
{[32.01]}\end{array}$ & $<0.05$ & NS & NS \\
\hline $\begin{array}{l}\text { Zuckerman } \\
\text { et al. } \\
(2004)^{25}\end{array}$ & $\begin{array}{l}\text { Patient profile } \\
+ \text { educational } \\
\text { material }\end{array}$ & $\begin{array}{l}\text { Estimated savings } \\
\text { of beta-blocker use } \\
7 \text { days post- } \\
\text { discharge (\$) }\end{array}$ & \multicolumn{9}{|c|}{71,970} \\
\hline & & $\begin{array}{l}\text { Estimated savings } \\
\text { of beta-blocker use } \\
30 \text { days post- } \\
\text { discharge }(\$)\end{array}$ & \multicolumn{9}{|c|}{76,678} \\
\hline
\end{tabular}

to prescribing patterns as compared with nearly $86 \%$ that used quasi-experimental or other nonrandomized controlled designs.

Commonly, a change in prescribing was defined by the presence or absence of a medication claim or dose change of a target agent. Such changes were often used as surrogate markers for improvements in health care and/or economic outcomes. While claims data can provide information regarding change in prescribing, it is not necessarily predictive of impact on longterm clinical or economic outcomes. Few programs attempted to evaluate impact on health care or economic outcomes, and ones that did often reported trends that failed to achieve statistical significance; a finding that was also observed by Hennessy et al. (2003). ${ }^{35}$ With the exception of one program, utilization tended to trend downward. Sleath et al. reported an interesting finding in their physician-only intervention group: an increase health care utilization (and associated costs) during the post-intervention period. ${ }^{22}$ This finding demonstrates that it is possible for an intervention to prompt an initial increase in utilization as additional medications and prescriber services may be required to address issues brought to light through intervention materials.

The lack of statistical significance observed for economic outcomes may be due in part to the relatively short followup periods used by the majority of identified programs. Additionally, the fact that interventions are based on individual transactions makes it difficult to quantify outcomes that may have been influenced by external circumstances outside the control of their program. It is possible that changes in hard outcomes may occur over longer periods of time; however, this brings into question the duration of impact following cessation of mailed intervention programs. Meyer et al. reported the effects of their one-time intervention mailing decreased to a point of nonsignificance after 6 months and continued to trend downwards at 12 months post-intervention. ${ }^{17}$ Though many others have reported significant effects at 6 and 12 months post-intervention, none evaluated the impact beyond 12 months of final intervention mailing. Several programs that provided continuous well-constructed mailings over an extended period (i.e., 12 months) achieved and maintained significant results throughout the entire intervention period. ${ }^{2-4,16}$ Such a program may be well equipped to evaluate economic outcomes.

This review identified several key factors that may contribute to intervention success, perhaps the most important being provision of patient-identifying data. Eighty-four percent of reviewed programs that used this method reported a significant impact on prescribing patterns. Comparatively, programs that provided prescriber feedback alone or in combination with other nonpatient-identifying materials had a lower rate of success. Intuitively, one could see how supplying prescribers with a list of identified patients would be conducive to prompt 
incorporation of intervention recommendations. Acceptance of recommendations provided via prescriber feedback requires recipients to approach future patient visits with the results in mind. However, the need to address more critical issues during office visits may prohibit incorporation of intervention recommendations, particularly if intervention effect does, in fact, decrease over time. Alternatively, physicians could retrospectively review their patient populations to determine specific individuals who may benefit from therapeutic adjustment. While thorough, the time-consuming nature of this task may dissuade physicians and therefore prohibit the potential impact of prescriber feedback. Our review indicated that prescriber feedback may be most useful in intervention involving antibiotics or medications with abuse potential. . $6,27,30$ Overprescribing such agents is denounced by the medical community as well as much of the general population. This negative outlook may prompt physicians to reevaluate their prescribing habits if they appear to be elevated, particularly in comparison with their peers.

Other factors that may contribute to intervention success included identifying recipients based on prescribing habits rather than geographic location or participation in a health care network. Programs that mailed interventions to prescribers based solely on practice location typically had little to no impact. ${ }^{5,6,28,30,32,43-45}$ This is consistent with the findings by Figueiras et al. (2001), who concluded that interventions that were personalized to recipients were associated with a higher rate of success. ${ }^{34}$ If costs permit, addition of pharmacists and/or patients as recipients may further improve intervention success. Additionally, interventions that consisted of educational materials alone rarely had an impact on prescribing practice. . $4,44,50,51$

The majority of programs offered recipients generalized recommendations that could be applied to the entire target population. Two programs reported successful results of interventions that provided patient-specific recommendations: Each enlisted a multidisciplinary team of practitioners to conduct case-by-case evaluations and formulate recommendations. ${ }^{15,47}$ While both methods were associated with positive results, providing generalized recommendations appeared to be a more timely and cost-effective approach. Furthermore, Meyer et al. found no difference between interventions that provided generalized and patient-specific recommendations. ${ }^{17}$ Whether a generalized or patient-specific manner is employed, recommendations supported by widely accepted guidelines or literature may be considered most acceptable to intervention recipients. Additionally, creating a focused, strongly supported intervention may be more effective than attempting to address multiple concerns.

The biggest study limitation cited by the majority of the authors is the use of claims data to describe prescription and medical histories. Data entry errors at the dispensing pharmacy can result in a multitude of inconsistencies with regard to prescriber information, such as incorrect physician identification and incorrect/outdated prescriber addresses. Among interventions that provided a response form, between $9 \%$ and $23 \%$ of recipients indicated they had received an intervention letter regarding a patient that did not belong to them and/or a medication they did not prescribe. ${ }^{18,20,21,46,48}$ In addition to the potential for administrative errors, prescription claims histories assume dispensing of medications as surrogate markers for disease states and prescription use. Though such markers can certainly provide insight into a patient's therapeutic management, this method is subject to error, particularly if selection is based solely on a computerized analysis. While it is efficient to preselect patients based on surrogate markers, it cannot replace the clinical judgments of a pharmacist. Several programs sought to reduce this potential for error and conducted manual reviews of identified profiles to ensure correct patient selection. ${ }^{10,15,25}$ Despite these potential drawbacks, claims data remain a widely accepted method of measuring drug exposure.

\section{Limitations}

All publications were identified using a single search engine, and other methods were not used to identify unpublished works or reports from individual agencies. Nevertheless, the authors felt PubMED provided a reliable representation of the majority of the publications in this field. It should be noted that there may be a potential bias towards publication of positive results. In a retrospective evaluation of publication bias, Easterbrook et al. (1991) found studies with a positive impact were twice as likely to be published as compared with those shown to have no impact. ${ }^{52}$ A majority of the mailed intervention programs were funded by governmental agencies, many of which were developed in response to OBRA 90. ${ }^{1}$ Mandates from federal legislation and/or drug utilization review boards may have resulted in methodological flaws that prohibited reports from being considered for publication. Additionally, selective reporting of the results may also have been guided by the funding source, as programs do not want to be targeted for wasting resources on failed initiatives.

As stated by Figueiras et al., organizational differences between health care systems and individual/cultural differences among prescribing practitioners may limit the generalizability of results. ${ }^{34}$ This may be especially true for programs that were conducted in countries that offer universal health coverage, as recipients may have differing views of intervention providers (particularly if it is governmental in nature) or health care as a whole. This review did not account for the potential differences in objectives between governmental and commercialized mailed intervention programs. The level of heterogeneity between reviewed publications, particularly with regard to targeted populations, outcomes, and disease states, prohibits provision of definitive results. For this reason, the authors elected to describe reported findings and identify 
general patterns associated with program success. In doing so, the authors acknowledge that it is likely external factors (i.e., seasonal variations, nature of disease state, patient population, and pharmaceutical marketing) may have contributed to observed changes in prescribing behavior.

Many of the reviewed publications reported outcomes from programs conducted in the 1990s to early 2000s. It is likely that prescriber attitude towards pharmacists and/or pharmacy benefits provider recommendations may have evolved since that time. Additionally, the majority of reports cited "physician" or "prescribers" as the primary intervention recipient and therefore may not have taken the expanding role of midlevel prescribers into account. Similarly, many reports did not specify if interventions were sent to primary care practitioners or specialists. For this reason, this review referred to all prescriber recipients as "prescribers."

Both randomized controlled and nonrandomized controlled/observational reports were included in this review. Many of the reviewed publications followed a pre/post quasiexperimental design and therefore may be subject to regression to the mean. ${ }^{3,4,7-14,19,21,22,24,25,30,45,48,50,53}$ The authors recognize the potential drawbacks of including nonrandomized controlled trials; however, due to a lack of available literature, we elected to include all reports of mailed letter interventions. Even among the randomized controlled reports, methodological errors existed (i.e., short duration that does not account for seasonal variability in prescribing, unmatched baseline characteristics, or use of comparator rather than true control group). This is likely because mailed intervention programs are initiated (and funded) with the primary goal of changing prescribing patterns to all beneficiaries. Measuring impact of an intervention is often a secondary motive, and exclusion of beneficiaries to create a control group may be viewed unfavorably by funding entities. While inclusion of quasi-experimental and observational studies may impact internal validity, the authors hope this comprehensive review will prove beneficial in the development of future mailed intervention programs.

\section{Conclusions}

The results of this review indicate that a well-orchestrated letter intervention program has the potential to produce successful outcomes. Identifying recipients based on prescribing habits (as opposed to practice location), provision of recommendations that are supported by widely accepted clinical guidelines, inclusion of patient-identifying information, and addition of a second intervention recipient may have been associated with significant changes in prescribing behavior. Whether these changes translate into cost savings is unknown. Future research should focus on longer assessment periods, particularly for interventions that provide regular mailings over a period of months or years. Extended follow-up periods could also be used to determine if the impact of an intervention does in fact decrease over time. Though the funding source of mailed intervention programs may prohibit the use of randomized controlled designs, improvements in methodology would certainly improve the validity of study results. Additionally, future projects may wish to identify factors that prohibit prescriber acceptance of intervention recommendations from translating into actual changes in therapy.

\section{Authors}

MEI-JEN HO, PharmD, MSPH, is Assistant Professor of Pharmacotherapeutics and Clinical Research, College of Pharmacy, University of South Florida, Tampa, Florida, and JINEANE VENCI, PharmD, is a PGY-1 Community Pharmacy Resident, University of Rochester Medical Center, Rochester, New York.

AUTHOR CORRESPONDENCE: Mei-Jen Ho, PharmD, MSPH, University of South Florida College of Pharmacy, 12901 Bruce B. Downs Blvd., MDC 30, Tampa, FL 33612. Tel: 813.974.6333;

Fax: 813.905.9885; E-mail: mhol@health.usf.edu.

\section{DISCLOSURES}

The authors reported no funding for this manuscript and no financial or other potential conflicts of interest related to the subject matter of this manuscript. At the time of submission, Dr. Ho was Clinical Assistant Professor of Pharmacy Practice and Director of Retrospective Drug Utilization Review Program and Dr. Venci was Clinical Pharmacist at Retrospective Drug Utilization Review Program, School of Pharmacy and Pharmaceutical Sciences, University at Buffalo, Buffalo, New York.

Concept and design, data collection, and data interpretation, writing, and revisions were performed by both authors. Portions of this manuscript were presented as a poster abstract at the American Pharmacist Association's Annual Meeting and Exposition in New Orleans, Louisiana on March 10, 2012 .

\section{REFERENCES}

1. Omnibus Budget Reconciliation Act of 1990. U.S. Public Law 101-508. 101st Cong., 2d sess., January 5, 1990.

2. Fick DM, Maclean JR, Rodriguez NA, et al. A randomized study to decrease the use of potentially inappropriate medications among community-dwelling older adults in a southeastern managed care organization. Am J Manag Care. 2004;10(11 Pt 1):761-68.

3. Jing S, Naliboff A, Kaufman MB, Choy M. Descriptive analysis of mail interventions with physicians and patients to improve adherence with antihypertensive and antidiabetic medications in a mixed-model managed care organization of commercial and Medicare members. J Manag Care Pharm. 2011;17(5):355-66.

4. Kaufman MB, Brodin KA, Sarafian A. Effect of prescriber education on the use of medications contraindicated in older adults in a managed medicare population. J Manag Care Pharm. 2005;11(3):211-19.

5. Søndergaard J, Andersen M, Støvring H, Kragstrup J. Mailed prescriber feedback in addition to a clinical guideline has no impact: a randomised, controlled trial. Scand J Prim Health Care. 2003;21(1):47-51.

6. Søndergaard J, Andersen M, Vach K, Kragstrup J, Maclure M, Gram LF Detailed postal feedback about prescribing to asthma patients combined with a guideline statement showed no impact: a randomised controlled trial. Eur J Clin Pharmacol. 2002;58(2):127-32. 
7. Woodward MC, Streeton CL, Guttmann A, Killer GT, Peck RW. Polypharmacy management among Australian veterans: improving prescribing through the Australian Department of Veterans' Affairs' prescriber feedback programme. Intern Med J. 2008;38(2):95-100

8. Coleman CI, Reddy P, Laster-Bradley NM, Dorval S, Munagala B, White CM. Effect of practitioner education on adherence to asthma treatment guidelines. Ann Pharmacother. 2003;37(7-8):956-61.

9. Guo JJ, Gibson JT, Hancock GR, Barker KN. Retrospective drug utilization review and the behavior of Medicaid prescribers: an empirical marginal analysis. Clin Ther. 1995;17(6):1174-87.

10. Lee E, McNally DL, Zuckerman IH. Evaluation of a physicianfocused educational intervention on Medicaid children with asthma. Ann Pharmacother. 2004:38(6):961-66.

11. Zimmerman DR, Collins TM, Lipowski EE, Sainfort F. Evaluation of a DUR intervention: a case-study of histamine antagonists. Inquiry. 1994;31(1):89-101.

12. Zuckerman IH, Menis M, McNally DL, Layne BA, Mullins CD. Intervention for decreasing excessive acetaminophen use in Pennsylvania Medicaid recipients. Am J Health Syst Pharm. 2004;61(22):2410-14.

13. Collins TM, Mott DA, Bigelow WE, Zimmerman DR. A controlled letter intervention to change prescribing behavior: results of a dual-targeted approach. Health Serv Res. 1997;32(4):471-89.

14. Culbertson VL, Force RW, Cady PS, Force WS. Positive impact of a follow-up phone call to community pharmacies in a medicaid drug utilization program. Ann Pharmacother. 1999;33(5):541-47.

15. Groves RE. Therapeutic drug-use review for the Florida Medicaid Program. Am J Hosp Pharm. 1985;42(2):316-19.

16. Hoffman L, Enders JL, Pippins J, Segal R. Reducing claims for prescription drugs with a high potential for abuse. Am J Health Syst Pharm. 2003;60(4):371-74.

17. Meyer TJ, Van Kooten D, Marsh S, Prochazka AV. Reduction of polypharmacy by feedback to clinicians. J Gen Intern Med. 1991;6(2):133-36.

18. Okano GJ, Rascati KL. Effects of Medicaid drug utilization review intervention letters. Clin Ther. 1995;17(3):525-33.

19. Raisch DW, Sleath BL. Using feedback letters to influence the use of antiulcer agents in a Medicaid program. J Gen Intern Med. 1999;14(3):145-50.

20. Rascati KL, Okano GJ, Burch C. Evaluation of physician intervention letters. Med Care. 1996;34(8):760-66.

21. Seltzer JK, Kurt TL, Knodel LC, Dean B, Burch C. Drug utilization review of sedative/hypnotic agents in Texas Medicaid patients. Texas Medicaid Vendor Drug Program Drug Utilization Review Board. J Am Pharm Assoc (Wash). 2000;40(4):495-99.

22. Sleath B, Collins T, Kelly HW, McCament-Mann L, Lien T. Effect of including both physicians and pharmacists in an asthma drug-use review intervention. Am J Health Syst Pharm. 1997;54(19):2197-2200.

23. Smith DH, Christensen DB, Stergachis A, Holmes G. A randomized controlled trial of a drug use review intervention for sedative hypnotic medications. Med Care. 1998;36(7):1013-21.

24. Starner CI, Norman SA, Reynolds RG, Gleason PR. Effect of a retrospective drug utilization review on potentially inappropriate prescribing in the elderly. Am J Geriatr Pharmacother. 2009;7(1):11-19.

25. Zuckerman IH, Weiss SR, McNally D, Layne B, Mullins CD, Wang J. Impact of an educational intervention for secondary prevention of myocardial infarction on Medicaid drug use and cost. Am J Manag Care. 2004;10 (7 Pt 2):493-500

26. Anderson JF, McEwan KL, Hrudey WP. Effectiveness of notification and group education in modifying prescribing of regulated analgesics. CMAJ. 1996;154(1):31-39.
27. Hux JE, Melady MP, DeBoer D. Confidential prescriber feedback and education to improve antibiotic use in primary care: a controlled trial. CMAJ. 1999;161(4):388-92.

28. O'Connell DL, Henry D, Tomlins R. Randomised controlled trial of effect of feedback on general practitioners' prescribing in Australia. BMJ. 1999;318(7182):507-11

29. Pimlott NJG, Hux JE, Wilson LM, Kahan M, Li C, Rosser WW. Educating physicians to reduce benzodiazepine use by elderly patients: a randomized controlled trial. CMAJ. 2003;168(7):835-39.

30. Rokstad K, Straand J, Fugelli P. Can drug treatment be improved by feedback on prescribing profiles combined with therapeutic recommendations? A prospective, controlled trial in general practice. J Clin Epidemiol. 1995;48(8):1061-68.

31. Avorn J, Soumerai SB. Improving drug-therapy decisions through educational outreach. A randomized controlled trial of academically based "detailing." N Engl J Med. 1983;308(24):1457-63.

32. Naughton C, Feely J, Bennett K. A clustered randomized trial of the effects of feedback using academic detailing compared to postal bulletin on prescribing of preventative cardiovascular therapy. Fam Pract. 2007;24(5):475-80.

33. Davis RS, Bukstein DA, Luskin AT, Kailin JA, Goodenow G. Changing physician prescribing patterns through problem-based learning: an interactive, teleconference case-based education program and review of problembased learning. Ann of Allergy Asthma Immunol. 2004;93(3):237-42.

34. Figueiras A, Sastre I, Gestal-Otero JJ. Effectiveness of educational interventions on the improvement of drug prescription in primary care: a critical literature review. J Eval Clin Pract. 2001;7(2):223-41.

35. Hennessy S, Bilker WB, Zhou L, et al. Retrospective drug utilization review, prescribing errors, and clinical outcomes. JAMA. 2003;290(11):1494-99.

36. Lipton HL, Bird JA. Drug utilization review in ambulatory settings: state of the science and directions for outcomes research. Med Care. 1993;31(12):1069-82

37. Oxman AD, Thomson MA, Davis DA, Haynes RB. No magic bullets: a systematic review of 102 trials of interventions to improve professional practice. CMAJ. 1995;153(10):1423-31.

38. Bonds DE, Hogan PE, Bertoni AG, et al. A multifaceted intervention to improve blood pressure control: the Guideline Adherence for Heart Health (GLAD) study. Am Heart J. 2009;157(2):278-84.

39. Graham SD, Hartzema AG, Sketris IS, Winterstein AG. Effect of an academic detailing intervention on the utilization rate of cyclooxygenase-2 inhibitors in the elderly. Ann Pharmacother. 2008;42(6):749-56.

40. Simon SR, Majumdar SR, Prosser LA, et al. Group versus individual academic detailing to improve the use of antihypertensive medications in primary care: a cluster-randomized controlled trial. Am J Med. 2005;118(5):521-28.

41. Soumerai SB, Avorn J. Economic and policy analysis of university-based drug "detailing." Med Care. 1986;24(4):313-31.

42. Zillich AJ, Ackermann RT, Stump TE, et al. An evaluation of educational outreach to improve evidence-based prescribing in Medicaid: a cautionary tale. J Eval Clin Pract. 2008;14(5):854-60.

43. Vægter K, Wahlström R, Wedel H, Svärdsudd K. Effect of mailed feedback on drug prescribing profiles in general practice: a seven-year longitudinal study in Storstrøm County, Denmark. Ups J Med Sci. 2010;115(4):238-44.

44. Dormuth CR, Maclure M, Bassett K, Jauca C, Whiteside C, Wright JM. Effect of periodic letters on evidence-based drug therapy on prescribing behaviour: a randomized trial. CMAJ. 2004;171(9):1057-61.

45. Schectman JM, Kanwal NK, Schroth WS, Elinsky EG. The effect of an education and feedback intervention on group-model and network-model health maintenance organization physician prescribing behavior. Med Care. 1995;33(2):139-44. 
46. Coleman CI, Reddy P, Laster-Bradley NM, Dorval S, Munagala B, White CM. Prescriber and pharmacist responses to intervention letters for Connecticut Medicaid beneficiaries with asthma. Am J Health Syst Pharm. 2003;60(11):1142-44.

47. Allard J, Hebert R, Rioux M, Asselin J, Voyer L. Efficacy of a clinical medication review on the number of potentially inappropriate prescriptions prescribed for community-dwelling elderly people. CMAJ. 2001;164(9):1291-96.

48. Sleath B, Collins T. Physician responses to an educational intervention on improving their long-term prescribing of sedatives. Patient Educ Couns. 1997;31(3):215-22.

49. Bjornson DC, Rector TS, Daniels CE, Wertheimer AI, Snowdon DA, Litman TJ. Impact of a drug-use review program intervention on prescribing after publication of a randomized clinical trial. Am J Hosp Pharm. 1990;47(7):1541-46

50. Owens C, Pugmire B, Owens K. A migraine prophylaxis educational intervention in a Medicaid population. Headache. 2008;48(2):267-71.
51. Shatin D, Gardner JS, Stergachis A, Blough D, Graham D. Impact of mailed warning to prescribers on the co-prescription of tramadol and antidepressants. Pharmacoepidemiol Drug Saf. 2005;14(3):149-54.

52. Easterbrook PJ, Berlin JA, Gopalan R, Matthews DR. Publication bias in clinical research. Lancet. 1991;337(8746):867-72.

53. Stephenson J, Imrie J. Why do we need randomised controlled trials to assess behavioural interventions? BMJ. 1998;316(7131):611-13.

54. Yusuf S, Peto R, Lewis J, Collins R, Sleight P. Beta blockade during and after myocardial infarction: an overview of the randomized trials. Prog Cardiovasc Dis. 1985;27(5):335-71.

55. Régie de l'assurance-maladie du Québec. Rapport du Comité d'analyse de la consommation médicamenteuse des personnes âgées. Québec (QC): The Gouvernement de Québec; 1992.

56. Beers MH. Explicit criteria for determining potentially inappropriate medication use by the elderly. An update. Arch Intern Med. 1997:157(14):1531-36. 